CRYSTALLOGRAPHIC COMMUNICATIONS

ISSN 2056-9890

Received 4 June 2021

Accepted 18 June 2021

Edited by J. Ellena, Universidade de Sâo Paulo, Brazil

Keywords: X-ray structure; ruthenium(II); rhenium(II); PTA; magnetism; crystal structure.

CCDC reference: 2075886

Supporting information: this article has supporting information at journals.iucr.org/e

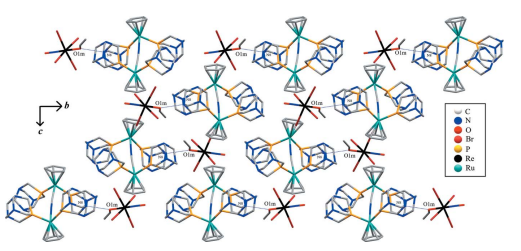

OPEN $\odot$ ACCESS

\section{Crystal structure and magnetic study of the complex salt $\left[\mathrm{RuCp}(\mathrm{PTA})_{2}-\mu-\mathrm{CN}-1 \kappa \mathrm{C}: 2 \kappa \mathrm{N}-\mathrm{RuCp}(\mathrm{PTA})_{2}\right]-$ $\left[\operatorname{Re}(\mathrm{NO}) \mathrm{Br}_{4}(\mathrm{EtOH})_{0.5}(\mathrm{MeOH})_{0.5}\right]$}

\author{
Mario Pacheco, ${ }^{\mathrm{a} *}$ Natalia Alvarez, ${ }^{\mathrm{a}}$ Alicia Cuevas, ${ }^{\mathrm{a}}$ Antonio Romerosa, ${ }^{\mathrm{b}}$ Francesc \\ Lloret $^{\mathrm{c}}$ and Carlos Kremer ${ }^{\mathrm{a}}$
}

aArea Quimica Inorganica, Facultad de Quimica, Universidad de la República, 11800, Montevideo, Uruguay, barea de
Quimica Inorganica-CIESOL, Universidad de Almeria, 04120 Almeria, Spain, and 'Instituto de Ciencia Molecular,
Universidad de Valencia, C/ Catedratico Jose, Beltran 2, 46980 Paterna, Valencia, Spain. *Correspondence e-mail:
mpacheco@fq.edu.uy

A new $\mathrm{Ru}^{\mathrm{II}}-\mathrm{Re}^{\mathrm{II}}$ complex salt, $\mu$-cyanido- $\kappa^{2} C: N$-bis[ $\left[\eta^{5}\right.$-cyclopentadienyl)bis(3,5,7-triazaphosphaadamantane- $\kappa P$ )ruthenium(II)] tetrabromido(ethanol/ methanol- $\kappa O$ )nitrosylrhenate(II), $\left[\mathrm{Ru}(\mathrm{CN})\left(\mathrm{C}_{5} \mathrm{H}_{5}\right)_{2}\left(\mathrm{C}_{6} \mathrm{H}_{12} \mathrm{~N}_{3} \mathrm{P}\right)_{4}\right]\left[\mathrm{ReBr}_{4}(\mathrm{NO})-\right.$ $\left.\left(\mathrm{CH}_{4} \mathrm{O}\right)_{0.5}\left(\mathrm{C}_{2} \mathrm{H}_{6} \mathrm{O}\right)_{0.5}\right] \quad$ or $\quad\left[\mathrm{RuCp}(\mathrm{PTA})_{2}-\mu-\mathrm{CN}-1 \kappa C: 2 \kappa^{2} N-\mathrm{RuCp}(\mathrm{PTA})_{2}\right]-$ $\left[\mathrm{Re}(\mathrm{NO}) \mathrm{Br}_{4}(\mathrm{EtOH})_{0.5}(\mathrm{MeOH})_{0.5}\right] \quad(\mathrm{PTA}=3,5,7$-triazaphosphaadamantane $)$ was obtained and characterized by single-crystal X-ray diffraction, elemental analysis and infrared spectroscopy. The title salt was obtained by liquid-liquid diffusion of methanol/DMSO solutions of $\left(\mathrm{NBu}_{4}\right)\left[\mathrm{Re}(\mathrm{NO}) \mathrm{Br}_{4}(\mathrm{EtOH})\right]$ and $\left[(\mathrm{PTA})_{2} \mathrm{CpRu}-\mu-\mathrm{CN}-1 \kappa C: 2 \kappa^{2} N-\mathrm{RuCp}(\mathrm{PTA})_{2}\right]\left(\mathrm{CF}_{3} \mathrm{SO}_{3}\right)$. The $\mathrm{Ru}^{\mathrm{II}}$ and $\mathrm{Re}^{\mathrm{II}}$ independent moieties correspond to a binuclear and mononuclear complex ion, respectively. A deep geometrical parameter analysis was performed, and no significant differences were found with earlier reports containing similar molecules. The magnetic properties were investigated in the temperature range $2.0-300 \mathrm{~K}$, and the complex behaves as a quasi-magnetically isolated spin doublet with weak antiferromagnetic interactions.

\section{Chemical context}

Ruthenium-arene-PTA (PTA = 3,5,7-triaza-phosphaadamantane) or RAPTA complexes are known in inorganic medicinal chemistry for their potent antitumor activity in vitro and in vivo, constituting a potential alternative to platinumbased drugs (Antonarakis \& Emadi, 2010; Gasser et al., 2011; Liang et al., 2017; Hey-Hawkins \& Hissler, 2019). Furthermore, PTA presents variable denticity allowing it to act as a versatile building block towards the synthesis of coordination polymers with applications in other areas such as chemical catalysis (Darensbourg et al., 1995; Scalambra et al., 2017; Scalambra, Lopez-Sanchez et al., 2020) and material science (Phillips et al., 2004). Professor Romerosa's group and coworkers have developed a family of water-soluble and airstable organometallic polymers containing an ' $\mathrm{RuCp}(\mathrm{PTA})_{2}$ ' $(\mathrm{Cp}=$ Cyclopentadienyl $)$ fragment. Most of them fit the general formula $\left[\left\{\mathrm{RuCp}(\mathrm{PTA})_{2}-\mu-\mathrm{CN}-1 \kappa C: 2 \kappa^{2} N\right.\right.$ $\left.\left.\mathrm{RuCp}(\mathrm{PTA})_{2}\right\}-\mu-M X_{m}\right]_{n}(M=\mathrm{Cd}, \mathrm{Ag}, \mathrm{Ni}, \mathrm{Au}, \mathrm{Co} ; X=$ halide or pseudohalide) (Serrano Ruiz et al., 2008; Lidrissi et al., 2005; Scalambra et al., 2015, 2018; Scalambra, Sierra-Martin et al., 2020). These polymers show exciting properties such as the formation of structured microparticles, amorphization under 
low pressures (Scalambra et al., 2015, 2016), the formation of layered structures that can be exfoliated in ultra-thin 3D layers (Scalambra, Sierra-Martin et al., 2020), the formation of gels in the presence of water (Sierra-Martin et al., 2018, 2019; Serrano Ruiz et al., 2008) or the capacity to capture water molecules in nanochannels (Scalambra et al., 2017). The described polymers include a wide variety of arrangements from one to three dimensions, and they may be classified as a new class of materials lying between metal-organic frameworks (MOFs) and infinite coordination polymers (ICPs) (Spokoyny et al., 2009). The preparation mostly involves the use of the bimetallic precursor $\mathrm{RuCp}(\mathrm{PTA})_{2}-\mu-\mathrm{CN}-$ $\left.1 \kappa C: 2 \kappa^{2} N-\mathrm{RuCp}(\mathrm{PTA})_{2}\right]\left(\mathrm{CF}_{3} \mathrm{SO}_{3}\right)$ in the reaction with other transition-metal cation salts or complexes, in an easy, robust and reproducible method (Serrano-Ruiz et al., 2014).

On top of that, rhenium nitrosyl complexes applications are widely recognized: catalysis, production of organonitrogen compounds, pollutant control, nitric oxide release drugs, assembly of devices with novel optical and magnetic properties, among other uses (Machura, 2005; Jiang et al., 2011; Probst et al., 2009; Ghosh et al., 2014; Dilworth, 2021). Kremer's group has performed a thorough magnetic study of a series of complexes $\left(\mathrm{NBu}_{4}\right)\left[\mathrm{Re}^{\mathrm{II}}(\mathrm{NO}) \mathrm{Br}_{4}(L)\right](L$ is an $N, O$ or $P$-donor neutral ligand) (Pacheco et al., 2013; Pacheco, Cuevas, González-Platas, Lloret et al., 2015). The low-spin outer $5 d^{5}$ shell results in strong spin-orbit interactions giving rise to a significant magnetic anisotropy, an essential feature for the potential construction of molecule-based magnets (Wang et al., 2011). In this work, we present the complex salt $\left[\mathrm{RuCp}(\mathrm{PTA})_{2}-\mu-\mathrm{CN}-1 \kappa C: 2 \kappa^{2} N-\mathrm{RuCp}(\mathrm{PTA})_{2}\right][\mathrm{Re}(\mathrm{NO})-$ $\left.\mathrm{Br}_{4}(\mathrm{EtOH})_{0.5}(\mathrm{MeOH})_{0.5}\right]$. The synthesis, single crystal X-ray crystal structure, and magnetic properties are discussed.

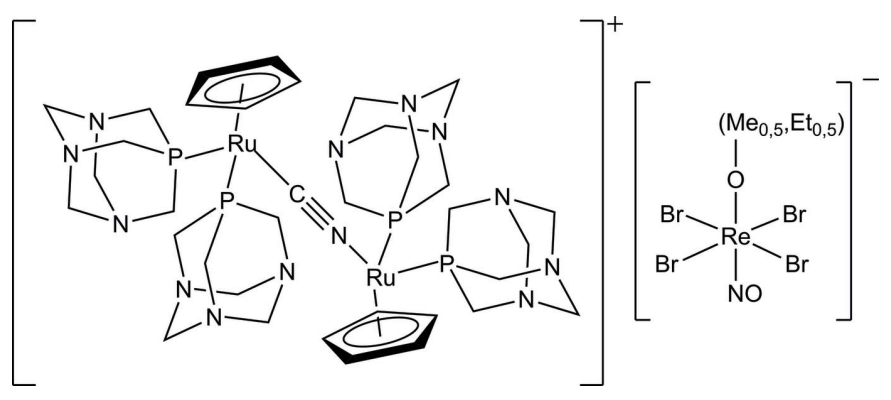

\section{Structural commentary}

The molecular structure of $\left[\mathrm{RuCp}(\mathrm{PTA})_{2}-\mu-\mathrm{CN}-1 \kappa C: 2 \kappa^{2} N\right.$ $\left.\mathrm{RuCp}(\mathrm{PTA})_{2}\right]\left[\operatorname{Re}(\mathrm{NO}) \mathrm{Br}_{4}(\mathrm{EtOH})_{0.5}(\mathrm{MeOH})_{0.5}\right]$ consists of discrete $\quad\left[\mathrm{RuCp}(\mathrm{PTA})_{2}-\mu-\mathrm{CN}-1 \kappa C: 2 \kappa^{2} N-\mathrm{RuCp}(\mathrm{PTA})_{2}\right]^{+}$ cations and $\left[\operatorname{Re}(\mathrm{NO}) \mathrm{Br}_{4}(\mathrm{EtOH})_{0.5}(\mathrm{MeOH})_{0.5}\right]^{-}$anions (Fig. 1), which coform the asymmetric unit.

The cation is an homobinuclear $\mathrm{Ru}^{\mathrm{II}}$ complex with two piano-stool fashion $\left\{\mathrm{RuCp}(\mathrm{PTA})_{2}\right\}$ moieties that are linked by $\mathrm{a}-\mathrm{CN}$ - bridging ligand. The $\left\{\mathrm{CpRu}(\mathrm{PTA})_{2}\right\}^{+}$moieties in each $\mathrm{Ru}_{2}$ unit exhibit a transoid arrangement related to the $\mathrm{Ru}-$ $\mathrm{C} \equiv \mathrm{N}-\mathrm{Ru}$ axis. The $\mathrm{Ru} 1-\mathrm{C} 25$ and $\mathrm{Ru} 2-\mathrm{N} 13$ distances are
Table 1

Hydrogen-bond geometry $\left(\AA{ }^{\circ}\right)$.

\begin{tabular}{lllll}
\hline$D-\mathrm{H} \cdots A$ & $D-\mathrm{H}$ & $\mathrm{H} \cdots A$ & $D \cdots A$ & $D-\mathrm{H} \cdots A$ \\
\hline $\mathrm{C} 10-\mathrm{H} 10 A \cdots \mathrm{Br} 3^{\mathrm{i}}$ & 0.97 & 3.12 & $3.944(12)$ & 143 \\
$\mathrm{C} 10-\mathrm{H} 10 B \cdots \mathrm{Br} 2$ & 0.97 & 2.83 & $3.709(10)$ & 150 \\
$\mathrm{C} 1-\mathrm{H} 1 B \cdots \mathrm{Br} 4^{\text {ii }}$ & 0.97 & 3.03 & $3.967(9)$ & 163 \\
$\mathrm{C} 7-\mathrm{H} 7 B \cdots \mathrm{N} 9^{\mathrm{iii}}$ & 0.97 & 2.59 & $3.309(11)$ & 131 \\
$\mathrm{C} 8-\mathrm{H} 8 A \cdots \mathrm{Br} 3^{\mathrm{i}}$ & 0.97 & 2.89 & $3.772(12)$ & 151 \\
$\mathrm{C} 4-\mathrm{H} 4 B \cdots \mathrm{Br} 3^{\mathrm{i}}$ & 0.97 & 3.10 & $4.062(10)$ & 169 \\
$\mathrm{C} 5-\mathrm{H} 5 A \cdots \mathrm{Br} 1^{\text {ii }}$ & 0.97 & 3.10 & $3.918(10)$ & 143 \\
$\mathrm{C} 18-\mathrm{H} 18 A \cdots \mathrm{N} 4^{\text {iv }}$ & 0.97 & 2.53 & $3.208(11)$ & 127 \\
$\mathrm{C} 18-\mathrm{H} 18 B \cdots \mathrm{Br} 2^{\mathrm{v}}$ & 0.97 & 2.92 & $3.858(9)$ & 163 \\
$\mathrm{C} 19-\mathrm{H} 19 B \cdots \mathrm{Br} 1^{\text {vi }}$ & 0.97 & 3.09 & $3.938(11)$ & 147 \\
$\mathrm{C} 22-\mathrm{H} 22 B \cdots \mathrm{Br} 1^{\text {vi }}$ & 0.97 & 3.00 & $3.861(10)$ & 148 \\
$\mathrm{C} 23-\mathrm{H} 23 A \cdots \mathrm{Br} 4^{\text {vii }}$ & 0.97 & 3.10 & $4.007(12)$ & 156 \\
$\mathrm{C} 24-\mathrm{H} 24 A \cdots \mathrm{Br} 3^{\text {vii }}$ & 0.97 & 2.98 & $3.799(11)$ & 143 \\
$\mathrm{O}_{1} 1 \mathrm{M}-\mathrm{H} 1 \mathrm{M} \cdots \mathrm{N} 8^{\mathrm{iii}}$ & 0.85 & 1.88 & $2.709(9)$ & 166 \\
$\mathrm{C} 1 \mathrm{E} B-\mathrm{H} 101 \cdots \mathrm{Br} 3$ & 0.97 & 2.80 & $3.527(13)$ & 132 \\
$\mathrm{C} 2 \mathrm{E}-\mathrm{H} 2 \mathrm{E} 3 \cdots \mathrm{N} 6^{\mathrm{i}}$ & 0.96 & 2.36 & $3.15(3)$ & 140 \\
\hline
\end{tabular}

Symmetry codes: (i) $-x,-y,-z$; (ii) $-x, y+\frac{1}{2},-z+\frac{1}{2}$; (iii) $x-1, y, z$; (iv) $x+1, y, z$; (v) $-x+1,-y,-z$; (vi) $-x+1, y+\frac{1}{2},-z+\frac{1}{2}$; (vii) $x+1, y+1, z$.

2.008 (7) and $2.030(8) \AA$, respectively. The $\mathrm{Ru}-\mathrm{CN}-\mathrm{Ru}$ arrangement is practically linear: $<(\mathrm{Ru} 1-\mathrm{C} 25-\mathrm{N} 13)=$ $175.5(7)^{\circ}$ and $<(\mathrm{C} 25-\mathrm{N} 13-\mathrm{Ru} 2)=176.3(7)^{\circ}$. The $\mathrm{C} \equiv \mathrm{N}$ bond length of the cyano group is 1.14 (1) $\AA$. The distances from the centroid of each $\mathrm{Cp}$ ligand to the respective ruthenium atom are $1.886 \AA$ ( $\mathrm{Cp}-\mathrm{Ru} 1)$ and $1.878(\mathrm{Cp}-\mathrm{Ru} 2)$. The $\mathrm{Ru}-\mathrm{P}_{\mathrm{PTA}}$ distances are in the range 2.243 (2)-2.281 (2) $\AA$, which is in agreement with those found in similar compounds.

The complex anion is constituted by an $\mathrm{Re}^{\mathrm{II}}$ atom and displays a distorted octahedral geometry formed by four bromide ions in the equatorial plane, one nitrogen atom from the nitrosyl ligand, and one oxygen atom from an - $\mathrm{OH}$ group in apical positions. The - $\mathrm{OH}$ group comes from a methanol or an ethanol molecule, both with an s.o.f. of 0.5. The O1M and $\mathrm{C} 1 \mathrm{E}$ atomic positions are the same for both the $\mathrm{MeOH}$ and the EtOH ligand. The $\mathrm{Re} 1-\mathrm{O} 1 \mathrm{~m}-\mathrm{Cle}$ angle is $128.3(6)^{\circ}$. The NO group is practically linear with an O101-N101-Re1 angle of $178.6(10)^{\circ}$. The three atoms are also aligned with the $\mathrm{O} 1 \mathrm{M}$ atom of the alcohol ligand, exhibiting a N101-Re1O1M angle of $178.9(3)^{\circ}$. The rhenium atom is shifted from the main plane of $\mathrm{Br}$ ligands towards the apical $\mathrm{NO}$ group by $0.157 \AA$.
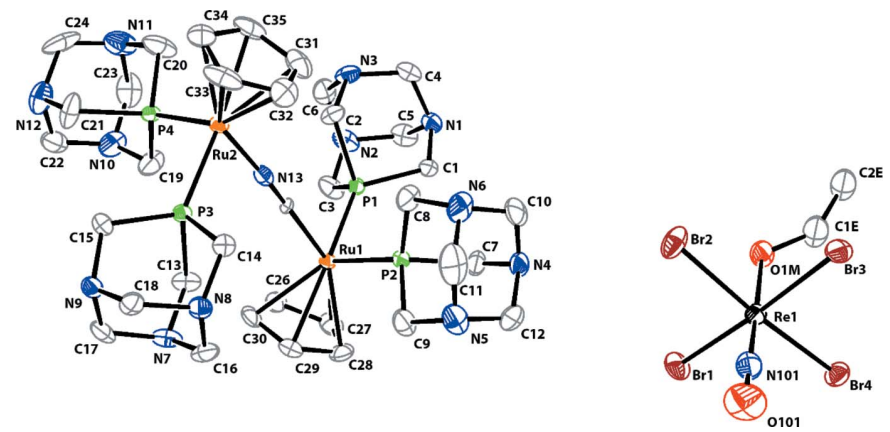

Figure 1

The asymmetric unit of the title compound, including atom labelling. Displacement ellipsoids are drawn at the $50 \%$ probability level. For clarity, $\mathrm{H}$ atoms have been omitted. 


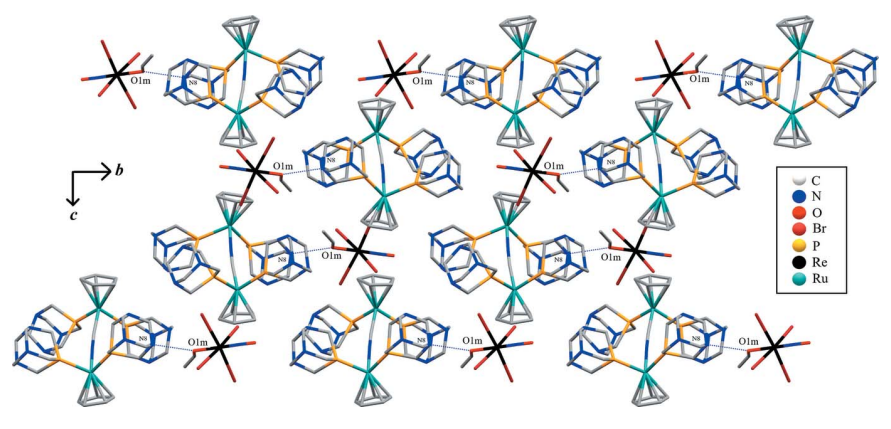

Figure 2

View along the $a$ axis of the title compound, with the $\mathrm{O} 1 \mathrm{M}-\mathrm{H} \cdots \mathrm{N} 8$ contacts (see Table 1 for details) represented by blue dashed lines. For clarity, $\mathrm{H}$ atoms have been omitted.

\section{Supramolecular features}

The complex crystallizes in the monoclinic $P 2_{1} / \mathrm{c}$ space group. The cations interconnect adjacent anions via $\mathrm{O}-\mathrm{H} \cdots \mathrm{N}$ hydrogen bonds and $\mathrm{C}-\mathrm{H} \cdots \mathrm{Br}$ interactions, forming an infinite three-dimensional framework (Table 1). The $\mathrm{O}-$ $\mathrm{H} \cdots \mathrm{N}$ interactions are given along the $b c$ plane and are defined by $\mathrm{O} 1 \mathrm{~m}$ as the donor atom from the $\mathrm{MeOH} / \mathrm{EtOH}$ ligand and $\mathrm{N} 8^{\mathrm{i}}$ atom from a PTA ligand at $(x-1, y, z)$ (Fig. 2). The $\mathrm{H} 1 \mathrm{M} \cdots \mathrm{N} 8^{\mathrm{i}}$ and $\mathrm{O} 1 \mathrm{M} \cdots \mathrm{N} 8^{\mathrm{i}}$ distances are 1.88 and 2.709 (9) $\AA$, respectively. The angle defined by O1M$\mathrm{H} 1 \mathrm{M} \cdots \mathrm{N} 8^{\mathrm{i}}$ is $165.5^{\circ}$.

The remaining hydrogen bonds are found between the PTA ligands from one cationic unit $\left[\mathrm{RuCp}(\mathrm{PTA})_{2}-\mu-\mathrm{CN}-\right.$ $\left.1 \kappa C: 2 \kappa^{2} N-\mathrm{RuCp}(\mathrm{PTA})_{2}\right]^{+}$and bromides from $\left[\mathrm{Re}(\mathrm{NO}) \mathrm{Br}_{4}{ }^{-}\right.$ $\left.(\mathrm{EtOH})_{0.5}(\mathrm{MeOH})_{0.5}\right]^{-}$units. The multiplicity and lack of defined directionality in the hydrogen-bond network are related to the fact that the major forces that stabilize the crystal are of electrostatic origin. The $\mathrm{C}-\mathrm{H} \cdots \mathrm{Br}$ and the $\mathrm{C} \cdots \mathrm{Br}$ distances range from $2.53-3.12 \AA$ and 3.208 (11)3.944 (12) $\AA$, respectively. The hydrogen-bond angle involving the $\mathrm{C}-\mathrm{H} \cdots \mathrm{Br}$ atoms vary between 127 and $169^{\circ}$. These geometrical values are in concordance with weak hydrogenbonding interactions (Desiraju, 1995; Metrangolo et al., 2006; Steed \& Atwood, 2009). The effect of the combined weak C$\mathrm{H} \cdots \mathrm{Br}$ bonds and their effect on the crystal assembly can be as significant as that of the strong interactions (Desiraju \& Steiner, 2001). The $\mathrm{C} 2 \mathrm{E}-\mathrm{H} \cdots \mathrm{N} 6$ bond is probably negligible because of the low energy expected for all $\mathrm{C}-\mathrm{H}$ bonds (Steed \& Atwood, 2009) and particularly considering the C2E 50\% atomic site occupation.

\section{Hirshfeld analysis}

To further understand the intermolecular interactions between the ionic complexes within the crystal structure, a Hirshfeld surface (Spackman \& Jayatilaka, 2009) was constructed around each ion. In addition, a 2D fingerprint plot analysis (Spackman \& McKinnon, 2002) was performed for each case. Crystal Explorer17 (Turner et al., 2017) was used to determine the surface and construct the plots. The Hirshfeld surfaces of both the anion and cation are illustrated in Fig. 3 (left) and 3 (right), respectively, showing surfaces that have been mapped over a $d_{\text {norm }}$ range of -0.6854 to 1.6426 a.u. (McKinnon et al., 2007). The color code employed for $d_{\text {norm }}$ is red for the shortest $d_{\text {norm }}$ and blue for the longest $d_{\text {norm }}$. Red spots in the surface correspond to the shortest contacts within the surface, indicating the formation of intermolecular bonds as those detailed in the previous section (supramolecular features).

The anion Hirshfeld surface shows how the most significant interaction is due to the $\mathrm{O} 1 \mathrm{~m}-\mathrm{H} \cdots \mathrm{N} 8$ bond, which is illustrated by bright-red spots in Fig. 3 (left), while the weaker spot corresponds to the $\mathrm{C} 2 \mathrm{E}-\mathrm{H} \cdots \mathrm{N} 6$ bond. What is more, the other minor red spots can be identified as $\mathrm{Br} \cdots \mathrm{H}$ interactions. These red spots (and thus the interionic interactions) can be correlated with the spikes observed in the twodimensional fingerprint plots. In fact, the anion fingerprint for all interactions exhibits characteristic spikes in the region $1.8 \AA<d_{\mathrm{i}}+d_{\mathrm{e}}<2.8 \AA$ resulting from $\mathrm{H} \cdots \mathrm{N}$ and $\mathrm{Br} \cdots \mathrm{H}$ interactions. There is a high-density area close to the $\mathrm{Br} \cdots \mathrm{H}$ spike, indicating a significant number of $\mathrm{Br} \cdots \mathrm{H}$ contacts in the crystal structure. In addition, the broad central spike extending up to the $\left(d_{\mathrm{i}}, d_{\mathrm{e}}\right)$ region of $(0.65 \AA, 0.78 \AA)$ reflects the significant amount of $\mathrm{H} \cdots \mathrm{H}$ contacts in the structure. Nevertheless, it is important to point out that the $\mathrm{H} \cdots \mathrm{H}$ contacts are usually difficult to localize in the Hirshfeld surface as they are spread all over the crystal packing. The Hirshfeld surface analysis for the cationic unit and its fingerprint also shows how $\mathrm{H} \cdots \mathrm{N}, \mathrm{N} \cdots \mathrm{H}, \mathrm{H} \cdots \mathrm{Br}$, and $\mathrm{H} \cdots \mathrm{H}$ contacts surround the $\left[\mathrm{RuCp}(\mathrm{PTA})_{2}-\mu-\mathrm{CN}-1 \kappa C: 2 \kappa^{2} N\right.$ $\mathrm{RuCp}(\mathrm{PTA})_{2}$ ] unit. The relative contributions of the different intermolecular contacts to the Hirshfeld area for both ions are shown in Fig. 4. In the anion, the major contributors ( $\sim 93 \%)$
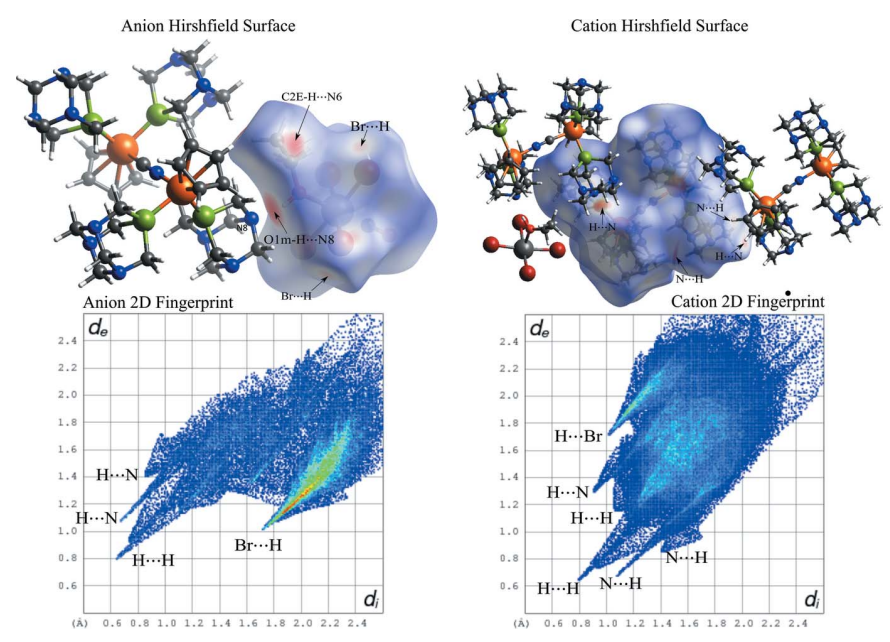

Figure 3

Projections of $d_{\text {norm }}$ mapped on Hirshfeld surfaces, showing the interactions between molecules and the two-dimensional $\left(d_{\mathrm{i}}, d_{\mathrm{e}}\right)$ fingerprint plot for the anionic unit $\left[\mathrm{Re}(\mathrm{NO}) \mathrm{Br}_{4}(\mathrm{EtOH})_{0.5}(\mathrm{MeOH})_{0.5}\right]^{-}$(left) and the cationic unit $\left[\mathrm{RuCp}(\mathrm{PTA})_{2}-\mu-\mathrm{CN}-1 \kappa C: 2 \kappa^{2} N-\mathrm{RuCp}(\mathrm{PTA})_{2}\right]^{+}$ (right). 


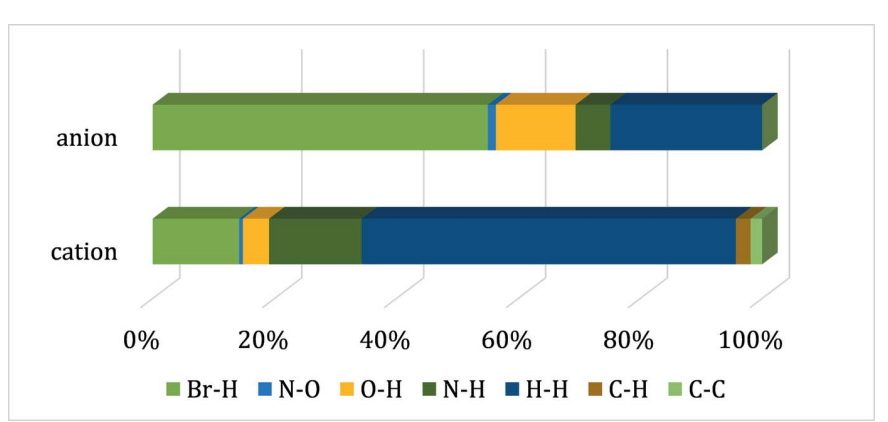

Figure 4

Relative contributions to Hirshfeld surface area for the close molecular contacts.

are from $\mathrm{Br} \cdots \mathrm{H}, \mathrm{O} \cdots \mathrm{H}$ and $\mathrm{H} \cdots \mathrm{H}$ contacts while in the cation, the Hirshfeld area is accounted mostly by the $\mathrm{Br} \cdots \mathrm{H}$, $\mathrm{N} \cdot \mathrm{H}$ and $\mathrm{H} \cdot \mathrm{H}$ contacts (over $90 \%$ ).

\section{Database survey}

A search in the Cambridge Structural Database (CSD) version 5.42 in the last update of February 2021 (Groom et al., 2016) for similar structures containing the anion and cation was performed. The $\left\{(\mathrm{PTA})_{2} \mathrm{CpRu}-\mu-\mathrm{CN}-\mathrm{RuCp}(\mathrm{PTA})_{2}\right\}$ moiety has been reported previously, once as an independent cationic unit in VOHCUS (Serrano-Ruiz et al., 2014) as well as a fragment within polynuclear polymeric structures CEQPEW (Scalambra et al., 2018), EDONET (Scalambra et al., 2016), GUVZUV (Scalambra, Sierra-Martin et al., 2020) and XADHES (Scalambra et al., 2015).

Regarding the anionic unit, examples of crystal structures containing tetrabromonitrosylrhenium(II) complexes are scarce. The CSD search yielded 19 hits. In all of them, the rhenium coordination sphere exhibits an octahedral geometry, with a practically lineal $\{\mathrm{Re}-\mathrm{NO}\}$ unit and a $\pi$-acceptor ligand such as phosphine or aromatic amines, usually coordinating trans- to the $-\mathrm{NO}$ group. The found $\pi$-acceptor ligands include: MeCN (Ciani et al., 1975), EtOH (Ciani et al., 1975), pyrazine (Pacheco et al., 2013, 2014; Pacheco, Cuevas, González-Platas, \& Kremer, 2015), nitrosyl (Mronga et al., 1982), tricyclohexylphosphine and triisopropylphosphine (Jiang et al., 2010), nicotinic acid and nicotinate anion (Pacheco, Cuevas, González-Platas, Lloret et al., 2015), pyridine, pyrimidine and pyridazine (Pacheco et al., 2013). All $\mathrm{Re}-\mathrm{Br}$ distances observed in the complex reported herein, as well as the $\mathrm{Re}-\mathrm{N}$ and $\mathrm{N}-\mathrm{O}$ distances found, agree with those found for previously reported structures (see Figs. 1-3 in the supporting information).

A search in the CSD for complexes containing a metal ion coordinating a $\mathrm{MeOH}$ molecule yielded 13705 structures with the $M-\mathrm{O}-\mathrm{C}$ angle lying in the range $123.333-130.865^{\circ}$ (without considering possible outlier values). The same angle for metals coordinating an EtOH is in the range 124.464$132.412^{\circ}$ (without considering possible outliers), in a total of 3503 reported structures. There are only five structures reported in the database containing ethanol coordinating to a rhenium atom, ABENRE (Ciani et al., 1975), PIXTOF
(Masood \& Hodgson, 1994), GEMVUR (Ikeda et al., 2012), EGAVEP (Hołyńska \& Lis, 2014) and PIMRAH (PinoCuevas et al., 2018). In those, the $\mathrm{Re}-\mathrm{O}-\mathrm{C}$ angles vary between 115.8 (4) and $135(1)^{\circ}$. The same search but for ReOHMe complexes yielded 15 structures, with the $\mathrm{Re}-\mathrm{O}-\mathrm{C}$ angles in the $121.232-133.389^{\circ}$ range. The only reported crystal structure in the CSD containing the $\left[\mathrm{Re}(\mathrm{NO}) \mathrm{Br}_{4}(\mathrm{EtOH})\right]^{-}$unit dates back to 1975 (ABENRE; Ciani et al., 1975). On the other hand, this is the first report of a crystal structure evidencing the coordination of a methanol molecule substituting ethanol.

Given that $\mathrm{C}-\mathrm{H} \cdots \mathrm{Br}$ bonds account for a significant fraction of intermolecular contacts, as seen in section 4 , a search was conducted involving this bonding scheme to check if the values presented in this article are within the bin frequently encountered in transition-metal compounds. The search restrained metal-Br $\cdots H$ distances to be lower than the sum of the vdW radius $(\sim 3.5 \AA)$. Compounds containing a $\mathrm{C}-\mathrm{Br} \cdots \mathrm{H}$ angle of less than $90^{\circ}$ were discarded, as the hydrogen atom in the hydrogen bond must not point away from the acceptor atom (Aakeröy et al., 1999). The search resulted in 36099 hits from 12143 structures. The histograms of $\mathrm{C} \cdots \mathrm{Br}$ distances and $\mathrm{C}-\mathrm{H} \cdots \mathrm{Br}$ angles (Figs. 4 and 5 in the supporting information) confirm that these $\mathrm{H} \cdots \mathrm{Br}$ contacts, considering the distance/angle criteria, can be identified as hydrogen bonds (Aakeröy et al., 1999; Metrangolo et al., 2006; Shimpi et al., 2007; Zhang et al., 2008).

\section{Magnetic measurements}

Magnetic susceptibility measurements on polycrystalline samples were carried out with a Superconducting Quantum Interference Design (SQUID) magnetometer in the temperature range $2.0-300 \mathrm{~K}$. In order to avoid saturation phenomena, we used external $d c$ magnetic fields of $500 \mathrm{G}$

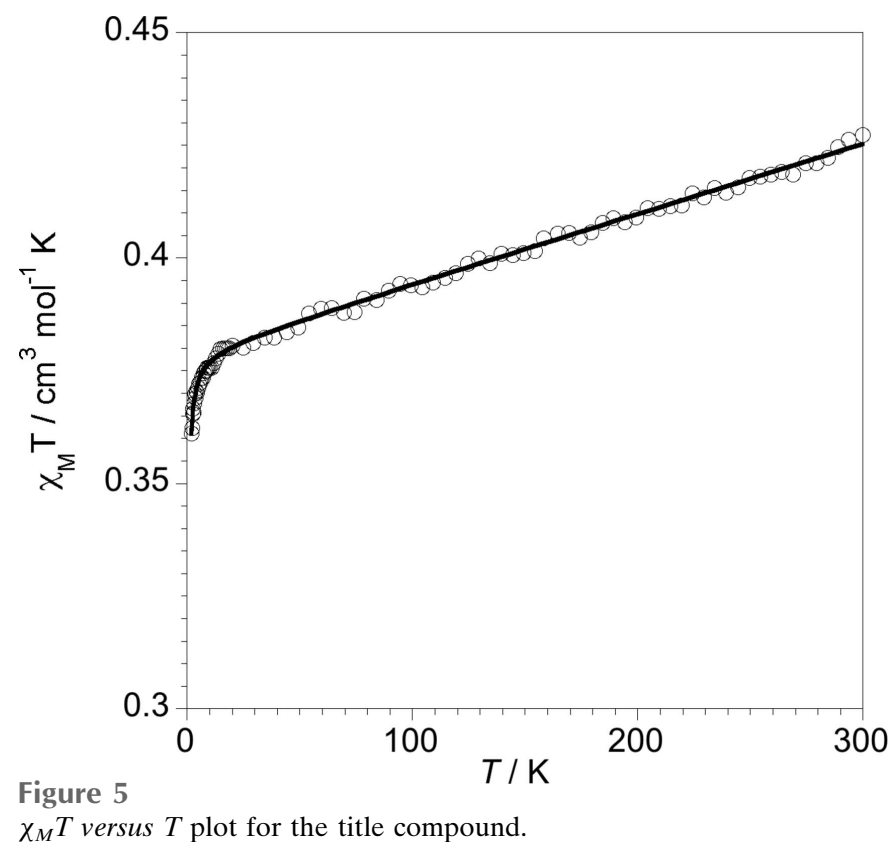


$(T<20 \mathrm{~K})$ and $5000 \mathrm{G}(T \geq 50 \mathrm{~K})$. Experimental susceptibilities were carefully corrected for the diamagnetism of the holder (gelatine capsule) and constituent atoms by applying Pascal's constants.

The magnetic behaviour of $\left[\mathrm{RuCp}(\mathrm{PTA})_{2}-\mu-\mathrm{CN}-\right.$ $\left.1 \kappa C: 2 \kappa^{2} N-\operatorname{RuCp}(\mathrm{PTA})_{2}\right]\left[\operatorname{Re}(\mathrm{NO}) \mathrm{Br}_{4}(\mathrm{EtOH})_{0.5}(\mathrm{MeOH})_{0.5}\right]$ is shown in Fig. 5 in the form of a $\chi_{M} T$ versus $T$ plot where $\chi_{M}$ is the molar magnetic susceptibility per one $\mathrm{Re}^{\mathrm{II}}$ ion and $T$ the absolute temperature. As expected, a straight line is observed for this compound (Pacheco et al., 2013). The thermal dependence of $\chi_{M} T$ is in line with one unpaired electron $(S=$ $\frac{1}{2}$ ) and a temperature independent paramagnetic contribution (TIP). The $\chi_{M} T$ value at room temperature is higher than that expected for an $S=\frac{1}{2}$ with $g=2.0\left(0.375 \mathrm{~cm}^{3} \mathrm{~K} \mathrm{~mol}^{-1}\right)$ due to the temperature-independent paramagnetism (TIP). The slight decrease below $10 \mathrm{~K}$ must be attributed to very weak intermolecular antiferromagnetic (AF) interactions between the $\left[\operatorname{Re}(\mathrm{NO}) \mathrm{Br}_{4}(\mathrm{EtOH})_{0.5}(\mathrm{MeOH})_{0.5}\right]^{-}$anions.

In this sense, we use equation (1), with $S=\frac{1}{2}$, to fit the experimental data.

$$
\chi_{M}=\frac{N \beta^{2} g^{2}}{3 k(T-\theta)} S(S+1)(1)
$$

Best-fit parameters were $g=2.01(1), T I P=155$ (3) $10^{-6}$ $\mathrm{cm}^{3} \mathrm{~mol}^{-1}$ and $\theta=-0.100$ (1) K. The calculated $g$ and TIP values are very close to those observed for similar complexes previously reported (Pacheco et al., 2013; Pacheco, Cuevas, González-Platas, Lloret et al., 2015). However, the Weiss parameter (intermolecular antiferromagnetic interaction), $\theta$, is lower, indicating that the paramagnetic anion is much more isolated, probably due to the vast diamagnetic counter-ion.

\section{Synthesis and crystallization}

\subsection{Experimental details}

$\left(\mathrm{NBu}_{4}\right)\left[\mathrm{Re}(\mathrm{NO}) \mathrm{Br}_{4}(\mathrm{EtOH})\right]$ and $\left[\mathrm{RuCp}(\mathrm{PTA})_{2}-\mu-\mathrm{CN}-\right.$ $1 \kappa C: 2 \kappa^{2} N$ - $\left.\mathrm{RuCp}(\mathrm{PTA})_{2}\right]\left(\mathrm{CF}_{3} \mathrm{SO}_{3}\right)$ were prepared as previously reported (Pacheco et al., 2013; Serrano-Ruiz et al., 2014). Solvents employed in the synthesis were purchased from commercial sources and used without further purification. Elemental analyses $(\mathrm{C}, \mathrm{H}, \mathrm{N}, \mathrm{S})$ were performed using a Flash 2000 (Thermo Scientific) elemental analyser. The IR spectra were recorded as $1 \% \mathrm{KBr}$ pellets on FTIR Shimadzu Prestige-21 spectrophotometer in the range $4000-400 \mathrm{~cm}^{-1}$.

\subsection{Synthesis}

A solution of $\left(\mathrm{NBu}_{4}\right)\left[\mathrm{Re}(\mathrm{NO}) \mathrm{Br}_{4}(\mathrm{EtOH})\right](0.012 \mathrm{mmol}$, $10 \mathrm{mg}$ ) dissolved in $5 \mathrm{~mL}$ of a methanol-DMSO (400:1, v/v) mixture was layered in an test tube with a solution of $\quad\left[\mathrm{RuCp}(\mathrm{PTA})_{2}-\mu-\mathrm{CN}-1 \kappa C: 2 \kappa^{2} N-\mathrm{RuCp}(\mathrm{PTA})_{2}\right]\left(\mathrm{CF}_{3} \mathrm{SO}_{3}\right)$ $(0.012 \mathrm{mmol}, 13 \mathrm{mg})$ in $5 \mathrm{~mL}$ of the same methanol-DMSO mixture; ca $5 \mathrm{~mL}$ of the solvent mixture should be added between the two reactant layers to decrease diffusion time. Deep reddish-brown plate-like crystals, suitable for single crystal X-ray diffraction were obtained after one week. The product was filtered and washed by decantation with methanol. Yield: $24 \%$. Analysis calculated for $\mathrm{Ru}_{2} \mathrm{C}_{36.5} \mathrm{~N}_{14-}$
Table 2

Experimental details.

Crystal data

Chemical formula

$M_{\text {r }}$

Crystal system, space group

Temperature (K)

$a, b, c(\AA)$

$\beta\left({ }^{\circ}\right)$

$V\left(\AA^{3}\right)$

Z

Radiation type

$\mu\left(\mathrm{mm}^{-1}\right)$

Crystal size (mm)

Data collection

Diffractometer

Absorption correction

$T_{\min }, T_{\max }$

No. of measured, independent and observed $[I>2 \sigma(I)]$ reflections

$R_{\text {int }}$

$(\sin \theta / \lambda)_{\max }\left(\AA^{-1}\right)$

$\left[\mathrm{Ru}(\mathrm{CN})\left(\mathrm{C}_{5} \mathrm{H}_{5}\right)_{2}\left(\mathrm{C}_{6} \mathrm{H}_{12} \mathrm{~N}_{3} \mathrm{P}\right)_{4}\right]_{2-}$

$\left[\mathrm{ReBr}_{4}(\mathrm{NO})\left(\mathrm{CH}_{4} \mathrm{O}\right)_{0.5^{-}}\right.$

$\left.\left(\mathrm{C}_{2} \mathrm{H}_{6} \mathrm{O}\right)_{0.5}\right]_{2}$

3123.73

Monoclinic, $P 2_{1} / c$

296

12.6027 (4), 17.7075 (6),

$23.0252(9)$

101.914 (1)

5027.7 (3)

2

Mo $K \alpha$

6.35

$0.48 \times 0.10 \times 0.03$

Refinement

$R\left[F^{2}>2 \sigma\left(F^{2}\right)\right], w R\left(F^{2}\right), S$

No. of reflections

No. of parameters

$\mathrm{H}$-atom treatment

$\Delta \rho_{\max }, \Delta \rho_{\min }\left(\mathrm{e} \AA^{-3}\right)$

Bruker D8 venture diffractometer Multi-scan (SADABS; Krause et al., 2015)

$0.485,0.751$

$56882,8565,6494$

0.079

0.589

Computer programs: APEX2 (Bruker, 2007), SAINT (Bruker, 2013), SHELXT2014/4 (Sheldrick, 2015a), SHELXL2018/3 (Sheldrick, 2015b), ORTEP-3 for Windows (Farrugia, 2012) and Mercury (Macrae et al., 2020).

$\mathrm{Re}_{1} \mathrm{O}_{2} \mathrm{Br}_{4} \mathrm{H}_{63} \mathrm{P}_{4}$ : C, 28.07; H, 4.07; N, 12.56; S. 0,00\%. Found: C, 27.18; H, 4.39; N, 12.53; S. 0,00\%. Selected IR absorption bands $\left(\mathrm{KBr}, \quad v_{\max } / \mathrm{cm}^{-1}\right): 3413\left[s, b r, v_{\mathrm{s}}(-\mathrm{OH})\right], 2922(w)$, $2114\left[m, v_{\mathrm{s}}(\mu-\mathrm{N} \equiv \mathrm{C})\right], 1759\left[s, v_{\mathrm{s}}(-\mathrm{NO})\right], 1280(m), 1242(\mathrm{~s})$, 1097(m), 1016(s), 970(s), 948(s), 833(w), 744(w), 574(m), $480(m)$.

\section{Refinement}

Crystal data, data collection and structure refinement details are summarized in Table 2. The $\mathrm{C}$-bound $\mathrm{H}$ atoms were included in calculated positions and treated as riding: $\mathrm{C}-\mathrm{H}$ distance between 0.94 and $0.98 \AA$ with $U_{\text {iso }}(\mathrm{H})=1.2 U_{\text {eq }}(\mathrm{C})$. Methanol/ethanol coordinating molecules were treated as positionally disordered utilizing the PART instruction with occupancy fixed to 0.5 applied to C1E, C1M, and C2E. C1M and $\mathrm{C} 1 \mathrm{E}$ were constrained to occupy equivalent positions. Meanwhile, C2E was located in the Fourier difference map and refined freely.

\section{Funding information}

Funding for this research was provided by: Programa de Desarrollo de las Ciencias Básicas (PEDECIBA) (grant to MP, NA, AC, CK); Comisión Sectorial de Investigación Científica (Apoyo a Grupos de Investigación No. 2003 to MP, AC, CK); Comisión Académica de Posgrado (CAP) 
(studentship to MP); University of Almeria (grant No. PPUENTE2020/011 to AR; grant No. PAI team FQM-317 to AR); Agencia Nacional de Investigación e Innovación (studentship to MP); Spanish MINECO (grant No. PID2019109735GB-I00; Unidad de Excelencia María de Maeztu CEX2019-000919-M); Generalitat Valenciana (grant No. AICO/2020/183).

\section{References}

Aakeröy, C. B., Evans, T. A., Seddon, K. R. \& Pálinkó, I. (1999). New J. Chem. 23, 145-152.

Antonarakis, E. S. \& Emadi, A. (2010). Cancer Chemother. Pharmacol. 66, 1-9.

Bruker (2007). APEX2. Bruker AXS Inc., Madison, Wisconsin, USA. Bruker (2013). SAINT Bruker AXS Inc., Madison, Wisconsin, USA. Ciani, G., Giusto, D., Manassero, M. \& Sansoni, M. (1975). J. Chem. Soc. Dalton Trans. pp. 2156-2161.

Darensbourg, D. J., Decuir, T. J. \& Reibenspies, J. H. (1995). Aqueous Organometallic Chemistry and Catalysis, Vol. edited by I. T. Horváth \& F. Joó, pp. 61-80. Dordrecht: Springer Netherlands.

Desiraju, G. R. (1995). Angew. Chem. Int. Ed. Engl. 34, 2311-2327.

Desiraju, G. R. \& Steiner, T. (2001). The Weak Hydrogen Bond in Structural Chemistry and Biology. Oxford University Press.

Dilworth, J. R. (2021). Coord. Chem. Rev. 436, 213822.

Farrugia, L. J. (2012). J. Appl. Cryst. 45, 849-854.

Gasser, G., Ott, I. \& Metzler-Nolte, N. (2011). J. Med. Chem. 54, 3-25.

Ghosh, S., Paul, S. S., Mitra, J. \& Mukherjea, K. K. (2014). J. Coord. Chem. 67, 1809-1834.

Groom, C. R., Bruno, I. J., Lightfoot, M. P. \& Ward, S. C. (2016). Acta Cryst. B72, 171-179.

Hey-Hawkins, E. \& Hissler, M. (2019). Smart Inorganic Polymers: Synthesis, Properties, and emerging applications in Materials and Life Sciences. Weinheim: Wiley-VCH.

Hołyńska, M. \& Lis, T. (2014). Inorg. Chim. Acta, 419, 96-104.

Ikeda, H., Yoshimura, T., Ito, A., Sakuda, E., Kitamura, N., Takayama, T., Sekine, T. \& Shinohara, A. (2012). Inorg. Chem. 51, 12065-12074.

Jiang, Y., Blacque, O. \& Berke, H. (2011). Dalton Trans. 40, 25782587.

Jiang, Y., Blacque, O., Fox, T., Frech, C. M. \& Berke, H. (2010). Chem. Eur. J. 16, 2240-2249.

Krause, L., Herbst-Irmer, R., Sheldrick, G. M. \& Stalke, D. (2015). J. Appl. Cryst. 48, 3-10.

Liang, J.-X., Zhong, H.-J., Yang, G., Vellaisamy, K., Ma, D.-L. \& Leung, C.-H. (2017). J. Inorg. Biochem. 177, 276-286.

Lidrissi, C., Romerosa, A., Saoud, M., Serrano-Ruiz, M., Gonsalvi, L. \& Peruzzini, M. (2005). Angew. Chem. Int. Ed. 44, 2568-2572.

Machura, B. (2005). Coord. Chem. Rev. 249, 2277-2307.

Macrae, C. F., Sovago, I., Cottrell, S. J., Galek, P. T. A., McCabe, P., Pidcock, E., Platings, M., Shields, G. P., Stevens, J. S., Towler, M. \& Wood, P. A. (2020). J. Appl. Cryst. 53, 226-235.

Masood, Md. A. \& Hodgson, D. J. (1994). Inorg. Chem. 33, 24882490.

McKinnon, J. J., Jayatilaka, D. \& Spackman, M. A. (2007). Chem. Commun. 3814-3816.
Metrangolo, P., Pilati, T. \& Resnati, G. (2006). CrystEngComm, 8, 946-947.

Mronga, N., Dehnicke, K. \& Fenske, D. (1982). Z. Anorg. Allg. Chem. 491, 237-244.

Pacheco, M., Cuevas, A., González-Platas, J., Faccio, R., Lloret, F., Julve, M. \& Kremer, C. (2013). Dalton Trans. 42, 15361-15371.

Pacheco, M., Cuevas, A., González-Platas, J., Gancheff, J. S. \& Kremer, C. (2014). J. Coord. Chem. 67, 4028-4038.

Pacheco, M., Cuevas, A., González-Platas, J. \& Kremer, C. (2015). Commun. Inorg. Synth. 2, 20-24.

Pacheco, M., Cuevas, A., González-Platas, J., Lloret, F., Julve, M. \& Kremer, C. (2015). Dalton Trans. 44, 11636-11648.

Phillips, A. D., Gonsalvi, L., Romerosa, A., Vizza, F. \& Peruzzini, M. (2004). Coord. Chem. Rev. 248, 955-993.

Pino-Cuevas, A., Graña, A., Abram, U., Carballo, R. \& VázquezLópez, E. M. (2018). CrystEngComm, 20, 4781-4792.

Probst, B., Kolano, C., Hamm, P. \& Alberto, R. (2009). Inorg. Chem. 48, 1836-1843.

Scalambra, F., López-Sánchez, B., Holzmann, N., Bernasconi, L. \& Romerosa, A. (2020). Organometallics, 39, 4491-4499.

Scalambra, F., Serrano-Ruiz, M., Gudat, D. \& Romerosa, A. (2016). ChemistrySelect, 1, 901-905.

Scalambra, F., Serrano-Ruiz, M. \& Romerosa, A. (2015). Macromol. Rapid Commun. 36, 689-693.

Scalambra, F., Serrano-Ruiz, M. \& Romerosa, A. (2017). Dalton Trans. 46, 5864-5871.

Scalambra, F., Serrano-Ruiz, M. \& Romerosa, A. (2018). Dalton Trans. 47, 3588-3595.

Scalambra, F., Sierra-Martin, B., Serrano-Ruiz, M., FernandezBarbero, A. \& Romerosa, A. (2020). Chem. Commun. 56, 94419444.

Serrano-Ruiz, M., Imberti, S., Bernasconi, L., Jadagayeva, N., Scalambra, F. \& Romerosa, A. (2014). Chem. Commun. 50, 11587-11590.

Serrano Ruiz, M., Romerosa, A., Sierra-Martin, B. \& FernandezBarbero, A. (2008). Angew. Chem. Int. Ed. 47, 8665-8669.

Sheldrick, G. M. (2015a). Acta Cryst. A71, 3-8.

Sheldrick, G. M. (2015b). Acta Cryst. C71, 3-8.

Shimpi, M. R., SeethaLekshmi, N. \& Pedireddi, V. R. (2007). Cryst. Growth Des. 7, 1958-1963.

Sierra-Martin, B., Serrano-Ruiz, M., García-Sakai, V., Scalambra, F., Romerosa, A. \& Fernandez-Barbero, A. (2018). Polymers, 10, 528.

Sierra-Martin, B., Serrano-Ruiz, M., Scalambra, F., FernandezBarbero, A. \& Romerosa, A. (2019). Polymers, 11, 1249.

Spackman, M. A. \& Jayatilaka, D. (2009). CrystEngComm, 11, 19-32.

Spackman, M. A. \& McKinnon, J. J. (2002). CrystEngComm, 4, 378392.

Spokoyny, A. M., Kim, D., Sumrein, A. \& Mirkin, C. A. (2009). Chem. Soc. Rev. 38, 1218-1227.

Steed, J. W. \& Atwood, J. L. (2009). Supramolecular Chemistry. Chichester: Wiley.

Turner, M. J., McKinnon, J. J., Wolff, S. K., Grimwood, D. J., Spackman, P. R., Jayatilaka, D. \& Spackman, M. A. (2017). CrystalExplorer17. University of Western Australia.

Wang, X.-Y., Avendaño, C. \& Dunbar, K. R. (2011). Chem. Soc. Rev. 40, 3213-3238.

Zhang, W., Tang, X., Ma, H., Sun, W.-H. \& Janiak, C. (2008). Eur. J. Inorg. Chem. pp. 2830-2836. 


\section{supporting information}

Acta Cryst. (2021). E77, 749-754 [https://doi.org/10.1107/S2056989021006381]

Crystal structure and magnetic study of the complex salt $\left[\mathrm{RuCp}(\mathrm{PTA})_{2}-\mu\right.$ -

$\left.\mathrm{CN}-1 \kappa \mathrm{C}: 2 \kappa \mathrm{N}-\mathrm{RuCp}(\mathrm{PTA})_{2}\right]\left[\mathrm{Re}(\mathrm{NO}) \mathrm{Br}_{4}(\mathrm{EtOH})_{0.5}(\mathrm{MeOH})_{0.5}\right]$

Mario Pacheco, Natalia Alvarez, Alicia Cuevas, Antonio Romerosa, Francesc Lloret and

\section{Carlos Kremer}

Computing details

Data collection: APEX2 (Bruker, 2007); cell refinement: APEX2 (Bruker, 2007); data reduction: SAINT (Bruker, 2013); program(s) used to solve structure: SHELXT2014/4 (Sheldrick, 2015a); program(s) used to refine structure:

SHELXL2018/3 (Sheldrick, 2015b); molecular graphics: ORTEP-3 for Windows (Farrugia, 2012) and Mercury (Macrae et al., 2020).

$\mu$-Cyanido- $\kappa^{2} C: N$-bis $\left[\left(\eta^{5}\right.\right.$-cyclopentadienyl) bis $(3,5,7$-triazaphosphaadamantane- $\kappa P)$ ruthenium(II)]

tetrabromido(ethanol/methanol- $\kappa O$ ) nitrosylrhenate(II)

Crystal data

$\left[\mathrm{Ru}(\mathrm{CN})\left(\mathrm{C}_{5} \mathrm{H}_{5}\right)_{2}\left(\mathrm{C}_{6} \mathrm{H}_{12} \mathrm{~N}_{3} \mathrm{P}\right)_{4}\right]_{2}\left[\mathrm{ReBr}_{4}(\mathrm{NO})\right.$

$\left.\left(\mathrm{CH}_{4} \mathrm{O}\right)_{0.5}\left(\mathrm{C}_{2} \mathrm{H}_{6} \mathrm{O}\right)_{0.5}\right]_{2}$

$M_{r}=3123.73$

Monoclinic, $P 2_{1} / c$

$a=12.6027(4) \AA$

$b=17.7075(6) \AA$

$c=23.0252(9) \AA$

$\beta=101.914(1)^{\circ}$

$V=5027.7(3) \AA^{3}$

$Z=2$

$F(000)=3036$

$D_{\mathrm{x}}=2.063 \mathrm{Mg} \mathrm{m}^{-3}$

Mo $K \alpha$ radiation, $\lambda=0.71073 \AA$

Cell parameters from 125 reflections

$\theta=3.1-16.9^{\circ}$

$\mu=6.35 \mathrm{~mm}^{-1}$

$T=296 \mathrm{~K}$

Prism, orange

$0.48 \times 0.10 \times 0.03 \mathrm{~mm}$

Data collection

Bruker D8 venture

$T_{\min }=0.485, T_{\max }=0.751$

diffractometer

Radiation source: sealed tube, SIEMENS

KFFMO2K-90C model 10190380

Curved graphite monochromator

Detector resolution: 10.4167 pixels $\mathrm{mm}^{-1}$

$\varphi$ and $\omega$ scans

Absorption correction: multi-scan

(SADABS; Krause et al., 2015)

56882 measured reflections

8565 independent reflections

6494 reflections with $I>2 \sigma(I)$

$R_{\text {int }}=0.079$

$\theta_{\text {max }}=24.7^{\circ}, \theta_{\min }=2.8^{\circ}$

$h=-14 \rightarrow 14$

$k=-20 \rightarrow 20$

$l=-27 \rightarrow 27$

Refinement

Refinement on $F^{2}$

Least-squares matrix: full

8565 reflections

$R\left[F^{2}>2 \sigma\left(F^{2}\right)\right]=0.044$

$w R\left(F^{2}\right)=0.118$

572 parameters

0 restraints

$S=0.99$

Primary atom site location: iterative 
Secondary atom site location: difference Fourier map

Hydrogen site location: mixed

$\mathrm{H}$-atom parameters constrained

$$
\begin{aligned}
& w=1 /\left[\sigma^{2}\left(F_{\mathrm{o}}{ }^{2}\right)+(0.0568 P)^{2}+32.4246 P\right] \\
& \text { where } P=\left(F_{\mathrm{o}}^{2}+2 F_{\mathrm{c}}^{2}\right) / 3 \\
& (\Delta / \sigma)_{\max }<0.001 \\
& \Delta \rho_{\max }=1.45 \mathrm{e}^{-3} \\
& \Delta \rho_{\min }=-1.34 \text { e } \AA^{-3}
\end{aligned}
$$

\section{Special details}

Geometry. All esds (except the esd in the dihedral angle between two 1.s. planes) are estimated using the full covariance

\begin{tabular}{|c|c|c|c|c|c|}
\hline & $x$ & $y$ & $z$ & $U_{\text {iso }} * / U_{\text {eq }}$ & Occ. $(<1)$ \\
\hline Re1 & $-0.05570(3)$ & $-0.18588(2)$ & $0.11322(2)$ & $0.03581(11)$ & \\
\hline Ru1 & $0.48233(5)$ & $0.25106(4)$ & 0.22334 & $0.02682(15)$ & \\
\hline $\mathrm{Ru} 2$ & $0.66214(5)$ & $0.27893(4)$ & $0.04193(3)$ & $0.02792(16)$ & \\
\hline $\mathrm{Br} 1$ & $0.04222(9)$ & $-0.12082(7)$ & $0.20632(4)$ & $0.0584(3)$ & \\
\hline $\mathrm{Br} 2$ & $0.04855(10)$ & $-0.10735(7)$ & $0.05355(5)$ & $0.0667(3)$ & \\
\hline $\mathrm{Br} 3$ & $-0.16673(10)$ & $-0.23895(6)$ & 0.01809 (4) & $0.0595(3)$ & \\
\hline $\mathrm{Br} 4$ & $-0.17972(8)$ & $-0.24939(6)$ & $0.17119(4)$ & 0.0518 & \\
\hline $\mathrm{P} 1$ & $0.35033(15)$ & $0.33600(12)$ & $0.18506(8)$ & $0.0268(4)$ & \\
\hline $\mathrm{P} 2$ & $0.40014(16)$ & $0.15060(12)$ & $0.17422(9)$ & $0.0318(5)$ & \\
\hline P3 & $0.79305(16)$ & $0.20690(12)$ & $0.09819(8)$ & $0.0279(4)$ & \\
\hline P4 & $0.72619(16)$ & $0.39303(12)$ & $0.07922(9)$ & $0.0294(4)$ & \\
\hline O101 & $0.0961(8)$ & $-0.3111(6)$ & $0.1265(5)$ & $0.110(4)$ & \\
\hline N1 & $0.1390(6)$ & $0.3853(4)$ & 0.1485 & $0.0434(18)$ & \\
\hline $\mathrm{N} 2$ & $0.2668(7)$ & $0.4759(5)$ & $0.2046(4)$ & $0.054(2)$ & \\
\hline N3 & $0.2747(6)$ & $0.4471(5)$ & $0.1013(4)$ & $0.050(2)$ & \\
\hline N4 & $0.2311(6)$ & 0.0505 (4) & 0.1466 & $0.055(2)$ & \\
\hline N6 & $0.3384(9)$ & $0.0693(5)$ & 0.0709 (4) & $0.072(3)$ & \\
\hline N7 & $0.8856(6)$ & $0.1427(4)$ & $0.2069(3)$ & $0.048(2)$ & \\
\hline N8 & $0.8650(5)$ & 0.0578 & $0.1213(3)$ & $0.0384(17)$ & \\
\hline N9 & $1.0030(5)$ & 0.1578 & 0.1354 & $0.0389(17)$ & \\
\hline N10 & $0.7525(8)$ & $0.5001(5)$ & $0.1692(4)$ & $0.063(2)$ & \\
\hline N11 & $0.6862(10)$ & 0.5468 & $0.0695(5)$ & $0.079(3)$ & \\
\hline N12 & $0.8770(8)$ & 0.5093 & $0.1015(5)$ & $0.068(3)$ & \\
\hline $\mathrm{C} 10$ & $0.2299(9)$ & $0.0593(6)$ & $0.0845(6)$ & $0.075(4)$ & \\
\hline $\mathrm{H} 10 \mathrm{~A}$ & 0.185518 & 0.102699 & 0.069690 & $0.090 *$ & \\
\hline $\mathrm{H} 10 \mathrm{~B}$ & 0.196148 & 0.015088 & 0.063481 & $0.090^{*}$ & \\
\hline $\mathrm{C} 11$ & $0.4070(11)$ & $0.0061(7)$ & $0.0987(7)$ & $0.088(4)$ & \\
\hline H11A & 0.379585 & -0.040178 & 0.078516 & $0.106^{*}$ & \\
\hline H11B & 0.479829 & 0.013789 & 0.092099 & $0.106^{*}$ & \\
\hline N13 & $0.5859(5)$ & $0.2744(4)$ & $0.1114(3)$ & $0.0363(16)$ & \\
\hline N101 & $0.0350(7)$ & $-0.2596(5)$ & $0.1217(4)$ & $0.057(2)$ & \\
\hline $\mathrm{C} 1$ & $0.2033(6)$ & $0.3182(5)$ & $0.1677(4)$ & $0.043(2)$ & \\
\hline $\mathrm{H} 1 \mathrm{~A}$ & 0.187494 & 0.280371 & 0.136628 & $0.052 *$ & \\
\hline H1B & 0.181946 & 0.297768 & 0.202646 & $0.052 *$ & \\
\hline
\end{tabular}
matrix. The cell esds are taken into account individually in the estimation of esds in distances, angles and torsion angles; correlations between esds in cell parameters are only used when they are defined by crystal symmetry. An approximate (isotropic) treatment of cell esds is used for estimating esds involving l.s. planes.

Fractional atomic coordinates and isotropic or equivalent isotropic displacement parameters $\left(\AA^{2}\right)$ 


\begin{tabular}{|c|c|c|c|c|}
\hline $\mathrm{C} 2$ & $0.3562(7)$ & $0.3865(5)$ & 0.1154 (4) & $0.044(2)$ \\
\hline $\mathrm{H} 2 \mathrm{~A}$ & 0.427904 & 0.408071 & 0.118761 & $0.053 *$ \\
\hline $\mathrm{H} 2 \mathrm{~B}$ & 0.344982 & 0.350473 & 0.082962 & $0.053^{*}$ \\
\hline $\mathrm{C} 3$ & $0.3459(8)$ & $0.4201(5)$ & 0.2319 (4) & 0.053 \\
\hline $\mathrm{H} 3 \mathrm{~A}$ & 0.329067 & 0.404229 & 0.269273 & $0.063 *$ \\
\hline H3B & 0.417142 & 0.443326 & 0.240483 & $0.063 *$ \\
\hline C7 & $0.2643(7)$ & $0.1199(6)$ & $0.1800(5)$ & $0.053(3)$ \\
\hline H7A & 0.262715 & 0.111570 & 0.221448 & $0.064 *$ \\
\hline H7B & 0.212869 & 0.159678 & 0.165259 & $0.064^{*}$ \\
\hline $\mathrm{C} 8$ & $0.3864(9)$ & $0.1426(6)$ & 0.0934 (4) & 0.057 (3) \\
\hline H8A & 0.340877 & 0.183277 & 0.074070 & $0.069 *$ \\
\hline H8B & 0.457243 & 0.147751 & 0.083554 & $0.069^{*}$ \\
\hline $\mathrm{C} 9$ & $0.4687(8)$ & $0.0597(5)$ & 0.1959 (6) & $0.064(3)$ \\
\hline H9A & 0.542754 & 0.062672 & 0.190220 & $0.077 *$ \\
\hline H9B & 0.471397 & 0.050991 & 0.237786 & $0.077 *$ \\
\hline N5 & $0.4139(8)$ & $-0.0044(5)$ & $0.1616(5)$ & $0.071(3)$ \\
\hline $\mathrm{C} 4$ & $0.1629(7)$ & $0.4158(6)$ & 0.0938 (4) & $0.048(2)$ \\
\hline $\mathrm{H} 4 \mathrm{~A}$ & 0.111021 & 0.455341 & 0.079097 & $0.057^{*}$ \\
\hline H4B & 0.153739 & 0.376066 & 0.064165 & $0.057^{*}$ \\
\hline $\mathrm{C} 5$ & $0.1567(8)$ & $0.4440(6)$ & 0.1932 (4) & $0.055(3)$ \\
\hline $\mathrm{H} 5 \mathrm{~A}$ & 0.142735 & 0.423527 & 0.229964 & $0.066^{*}$ \\
\hline H5B & 0.105007 & 0.484405 & 0.180683 & $0.066^{*}$ \\
\hline C6 & $0.2891(9)$ & $0.5031(5)$ & $0.1497(5)$ & $0.061(3)$ \\
\hline H6A & 0.363300 & 0.521225 & 0.156964 & $0.074^{*}$ \\
\hline H6B & 0.242057 & 0.545789 & 0.136630 & $0.074 *$ \\
\hline C12 & $0.3026(9)$ & $-0.0125(5)$ & $0.1703(5)$ & $0.064(3)$ \\
\hline H12A & 0.304399 & -0.016960 & 0.212516 & $0.077 *$ \\
\hline H12B & 0.272252 & -0.058858 & 0.151518 & $0.077^{*}$ \\
\hline $\mathrm{C} 13$ & $0.8036(8)$ & 0.1997 (5) & $0.1790(4)$ & $0.043(2)$ \\
\hline H13A & 0.823328 & 0.248627 & 0.196916 & $0.051 *$ \\
\hline H13B & 0.733433 & 0.186003 & 0.186840 & $0.051^{*}$ \\
\hline $\mathrm{C} 14$ & $0.7814(7)$ & $0.1043(5)$ & $0.0826(4)$ & $0.038(2)$ \\
\hline $\mathrm{H} 14 \mathrm{~A}$ & 0.710386 & 0.087306 & 0.087148 & $0.046^{*}$ \\
\hline H14B & 0.786260 & 0.095862 & 0.041597 & $0.046^{*}$ \\
\hline $\mathrm{C} 15$ & $0.9390(6)$ & $0.2153(5)$ & 0.0987 (4) & $0.0356(19)$ \\
\hline H15A & 0.950021 & 0.210954 & 0.058339 & $0.043 *$ \\
\hline H15B & 0.964059 & 0.264882 & 0.113423 & $0.043^{*}$ \\
\hline C16 & $0.8588(8)$ & $0.0680(5)$ & $0.1841(4)$ & 0.049 (2) \\
\hline H16A & 0.785748 & 0.056039 & 0.188513 & $0.058^{*}$ \\
\hline H16B & 0.907554 & 0.032285 & 0.207982 & $0.058^{*}$ \\
\hline $\mathrm{C} 17$ & $0.9918(7)$ & $0.1643(6)$ & 0.1968 (4) & 0.049 (2) \\
\hline H17A & 1.006169 & 0.216183 & 0.209553 & $0.059^{*}$ \\
\hline H17B & 1.046353 & 0.132840 & 0.221310 & $0.059 *$ \\
\hline C18 & $0.9748(7)$ & $0.0812(5)$ & 0.1149 (4) & $0.041(2)$ \\
\hline H18A & 1.027553 & 0.046580 & 0.137183 & $0.049 *$ \\
\hline H18B & 0.979171 & 0.077487 & 0.073475 & $0.049 *$ \\
\hline C19 & $0.7220(10)$ & $0.4204(6)$ & $0.1561(4)$ & $0.061(3)$ \\
\hline H19A & 0.649346 & 0.412212 & 0.162738 & $0.073 *$ \\
\hline
\end{tabular}




\begin{tabular}{|c|c|c|c|c|c|}
\hline H19B & 0.771012 & 0.388218 & 0.183392 & $0.073^{*}$ & \\
\hline $\mathrm{C} 20$ & $0.6489(11)$ & $0.4732(6)$ & $0.0421(5)$ & 0.077 (4) & \\
\hline $\mathrm{H} 20 \mathrm{~A}$ & 0.655078 & 0.473891 & 0.000761 & $0.092 *$ & \\
\hline H20B & 0.572964 & 0.466475 & 0.043119 & $0.092 *$ & \\
\hline $\mathrm{C} 21$ & $0.8624(9)$ & $0.4296(6)$ & $0.0794(6)$ & 0.069 & \\
\hline $\mathrm{H} 21 \mathrm{~A}$ & 0.915554 & 0.397600 & 0.104395 & $0.083^{*}$ & \\
\hline $\mathrm{H} 21 \mathrm{~B}$ & 0.875592 & 0.427327 & 0.039412 & $0.083^{*}$ & \\
\hline $\mathrm{C} 22$ & $0.8613(9)$ & $0.5136(6)$ & $0.1613(5)$ & $0.065(3)$ & \\
\hline $\mathrm{H} 22 \mathrm{~A}$ & 0.883435 & 0.563405 & 0.176712 & $0.078^{*}$ & \\
\hline H22B & 0.909056 & 0.477218 & 0.185063 & $0.078^{*}$ & \\
\hline $\mathrm{C} 23$ & $0.6783(10)$ & $0.5495(7)$ & $0.1312(6)$ & 0.075 (4) & \\
\hline $\mathrm{H} 23 \mathrm{~A}$ & 0.691530 & 0.600858 & 0.145481 & $0.090^{*}$ & \\
\hline H23B & 0.604921 & 0.536233 & 0.134178 & $0.090^{*}$ & \\
\hline $\mathrm{C} 24$ & $0.7978(14)$ & $0.5580(7)$ & $0.0637(5)$ & $0.087(5)$ & \\
\hline $\mathrm{H} 24 \mathrm{~A}$ & 0.817520 & 0.610183 & 0.073033 & $0.104^{*}$ & \\
\hline H24B & 0.802066 & 0.549376 & 0.022668 & $0.104 *$ & \\
\hline $\mathrm{C} 25$ & $0.5470(5)$ & $0.2690(4)$ & 0.1518 (3) & $0.0225(15)$ & \\
\hline $\mathrm{C} 26$ & $0.5553(8)$ & $0.3156(5)$ & 0.3068 (4) & $0.046(2)$ & \\
\hline $\mathrm{H} 26$ & 0.555150 & 0.370530 & 0.312146 & $0.055^{*}$ & \\
\hline $\mathrm{C} 27$ & $0.4748(8)$ & $0.2631(7)$ & $0.3181(4)$ & 0.053 & \\
\hline $\mathrm{H} 27$ & 0.409889 & 0.276314 & 0.333130 & $0.063^{*}$ & \\
\hline $\mathrm{C} 28$ & $0.5087(9)$ & $0.1890(6)$ & $0.3085(4)$ & $0.054(3)$ & \\
\hline $\mathrm{H} 28$ & 0.473025 & 0.142054 & 0.316367 & $0.065^{*}$ & \\
\hline $\mathrm{C} 29$ & $0.6103(7)$ & $0.1955(6)$ & $0.2917(4)$ & $0.045(2)$ & \\
\hline $\mathrm{H} 29$ & 0.656417 & 0.153412 & 0.284591 & $0.053 *$ & \\
\hline $\mathrm{C} 30$ & $0.6369(7)$ & $0.2727(6)$ & $0.2907(4)$ & 0.050 & \\
\hline $\mathrm{H} 30$ & 0.704052 & 0.292920 & 0.281675 & $0.060^{*}$ & \\
\hline $\mathrm{C} 31$ & $0.5113(8)$ & $0.2551(7)$ & $-0.0273(4)$ & $0.061(3)$ & \\
\hline H31 & 0.438182 & 0.249096 & -0.019477 & $0.074 *$ & \\
\hline $\mathrm{C} 32$ & $0.5831(10)$ & $0.1982(7)$ & $-0.0281(4)$ & $0.062(3)$ & \\
\hline H32 & 0.568991 & 0.144879 & -0.021077 & $0.075^{*}$ & \\
\hline $\mathrm{C} 33$ & $0.6779(10)$ & $0.2255(8)$ & -0.0420 & $0.068(4)$ & \\
\hline H33 & 0.739328 & 0.195217 & -0.048791 & $0.081 *$ & \\
\hline C34 & $0.6636(10)$ & $0.3038(8)$ & $-0.0516(4)$ & $0.074(4)$ & \\
\hline H34 & 0.713939 & 0.337921 & -0.065857 & $0.089 *$ & \\
\hline $\mathrm{C} 35$ & 0.5567 (9) & $0.3237(7)$ & $-0.0416(4)$ & $0.064(3)$ & \\
\hline H35 & 0.521157 & 0.373131 & -0.047466 & $0.077 *$ & \\
\hline $\mathrm{O} 1 \mathrm{M}$ & $-0.1667(5)$ & $-0.0925(4)$ & $0.1036(3)$ & $0.0546(17)$ & \\
\hline $\mathrm{H} 1 \mathrm{~m}$ & -0.145698 & -0.046869 & 0.108187 & $0.082 *$ & \\
\hline C1EB & $-0.2859(10)$ & $-0.0942(7)$ & $0.0884(6)$ & $0.076(3)$ & 0.5 \\
\hline H101 & -0.296961 & -0.144748 & 0.071928 & $0.091 *$ & 0.5 \\
\hline $\mathrm{H} 102$ & -0.301292 & -0.099033 & 0.127783 & $0.091 *$ & 0.5 \\
\hline $\mathrm{C} 2 \mathrm{E}$ & $-0.3839(19)$ & $-0.0593(14)$ & $0.0584(12)$ & $0.076(3)$ & 0.5 \\
\hline $\mathrm{H} 2 \mathrm{e} 1$ & -0.405709 & -0.021893 & 0.083727 & $0.114 *$ & 0.5 \\
\hline $\mathrm{H} 2 \mathrm{e} 2$ & -0.439669 & -0.096818 & 0.048511 & $0.114^{*}$ & 0.5 \\
\hline $\mathrm{H} 2 \mathrm{e} 3$ & -0.372188 & -0.035670 & 0.022699 & $0.114 *$ & 0.5 \\
\hline C1EA & $-0.2859(10)$ & $-0.0942(7)$ & $0.0884(6)$ & $0.076(3)$ & 0.5 \\
\hline H1eA & -0.319631 & -0.056188 & 0.058329 & $0.114^{*}$ & 0.5 \\
\hline
\end{tabular}




\begin{tabular}{llllll}
$\mathrm{H} 1 \mathrm{eB}$ & -0.320451 & -0.093614 & 0.121497 & $0.114^{*}$ & 0.5 \\
$\mathrm{H} 1 \mathrm{eC}$ & -0.314444 & -0.146063 & 0.065667 & $0.114^{*}$ & 0.5 \\
\hline
\end{tabular}

Atomic displacement parameters $\left(\AA^{2}\right)$

\begin{tabular}{|c|c|c|c|c|c|c|}
\hline & $U^{11}$ & $U^{22}$ & $U^{33}$ & $U^{12}$ & $U^{13}$ & $U^{23}$ \\
\hline $\operatorname{Re} 1$ & $0.0446(2)$ & $0.0355(2)$ & $0.02719(18)$ & $-0.00580(16)$ & $0.00707(14)$ & $-0.00453(14)$ \\
\hline Ru1 & $0.0259(3)$ & $0.0327(3)$ & $0.0212(3)$ & -0.0019 & $0.0033(2)$ & $0.0023(3)$ \\
\hline Ru2 & $0.0300(3)$ & 0.0349 (4) & $0.0180(3)$ & $0.0006(3)$ & $0.0031(2)$ & $-0.0007(3)$ \\
\hline $\mathrm{Br} 1$ & $0.0624(6)$ & $0.0696(7)$ & $0.0382(5)$ & $-0.0096(5)$ & $-0.0010(4)$ & $-0.0183(5)$ \\
\hline $\mathrm{Br} 2$ & $0.0934(8)$ & $0.0612(7)$ & $0.0558(6)$ & $-0.0312(6)$ & $0.0394(6)$ & $-0.0125(5)$ \\
\hline $\mathrm{Br} 3$ & $0.0914(8)$ & $0.0437(6)$ & $0.0345(5)$ & $-0.0140(5)$ & $-0.0078(5)$ & $-0.0047(4)$ \\
\hline $\mathrm{Br} 4$ & $0.0577(6)$ & $0.0529(6)$ & $0.0480(6)$ & $-0.0077(5)$ & $0.0184(4)$ & $0.0064(4)$ \\
\hline P1 & $0.0280(10)$ & $0.0299(11)$ & $0.0227(10)$ & $-0.0032(9)$ & $0.0056(8)$ & $-0.0018(8)$ \\
\hline P2 & $0.0303(10)$ & $0.0297(11)$ & $0.0330(11)$ & $-0.0023(9)$ & $0.0010(8)$ & $0.0038(9)$ \\
\hline P3 & $0.0303(10)$ & $0.0310(11)$ & $0.0222(10)$ & $0.0000(9)$ & $0.0054(8)$ & $0.0006(8)$ \\
\hline P4 & $0.0339(11)$ & $0.0292(11)$ & $0.0242(10)$ & $0.0016(9)$ & $0.0038(8)$ & $0.0049(8)$ \\
\hline O101 & $0.093(7)$ & $0.097(8)$ & $0.141(10)$ & $0.045(6)$ & $0.028(6)$ & $0.009(7)$ \\
\hline N1 & $0.037(4)$ & $0.046(5)$ & $0.044(4)$ & $0.003(4)$ & $0.002(3)$ & $0.003(4)$ \\
\hline $\mathrm{N} 2$ & $0.063(5)$ & $0.046(5)$ & $0.045(5)$ & $0.015(4)$ & $-0.005(4)$ & $-0.015(4)$ \\
\hline N3 & $0.056(5)$ & $0.047(5)$ & $0.051(5)$ & $0.014(4)$ & $0.018(4)$ & $0.022(4)$ \\
\hline N4 & $0.043(4)$ & $0.033(4)$ & $0.082(7)$ & -0.010 & -0.007 (4) & $0.010(4)$ \\
\hline N6 & $0.102(8)$ & $0.053(6)$ & $0.056(6)$ & $-0.038(6)$ & 0.007 (5) & $-0.019(5)$ \\
\hline N7 & $0.064(5)$ & $0.047(5)$ & $0.030(4)$ & 0.024 (4) & $0.006(3)$ & $0.006(3)$ \\
\hline N8 & $0.037(4)$ & $0.030(4)$ & $0.048(4)$ & $0.009(3)$ & 0.007 (3) & $0.003(3)$ \\
\hline N9 & $0.030(4)$ & $0.049(4)$ & $0.037(4)$ & $0.004(3)$ & $0.005(3)$ & 0.005 \\
\hline N10 & $0.091(7)$ & $0.053(6)$ & $0.047(5)$ & -0.018 & $0.018(5)$ & -0.010 \\
\hline N11 & $0.104(8)$ & $0.040(5)$ & $0.078(7)$ & $0.010(5)$ & $-0.013(6)$ & $0.008(5)$ \\
\hline N12 & $0.067(6)$ & $0.050(6)$ & $0.093(8)$ & $-0.018(5)$ & $0.032(5)$ & $-0.013(5)$ \\
\hline $\mathrm{C} 10$ & $0.072(8)$ & $0.048(7)$ & $0.087(9)$ & $-0.025(6)$ & $-0.029(7)$ & $-0.001(6)$ \\
\hline C11 & $0.083(9)$ & $0.055(8)$ & $0.127(13)$ & $-0.015(7)$ & $0.023(8)$ & $-0.051(8)$ \\
\hline N13 & $0.031(4)$ & $0.034(4)$ & $0.039(4)$ & $0.001(3)$ & -0.005 & -0.003 \\
\hline N101 & $0.053(5)$ & $0.062(6)$ & $0.054(5)$ & $-0.006(5)$ & $0.011(4)$ & -0.003 \\
\hline $\mathrm{C} 1$ & $0.032(4)$ & $0.050(6)$ & $0.046(5)$ & $0.000(4)$ & $0.002(4)$ & $0.018(4)$ \\
\hline $\mathrm{C} 2$ & $0.051(5)$ & $0.038(5)$ & $0.048(5)$ & 0.009 (4) & $0.022(4)$ & $0.012(4)$ \\
\hline $\mathrm{C} 3$ & $0.060(6)$ & $0.041(5)$ & $0.047(6)$ & $0.009(5)$ & $-0.011(5)$ & -0.020 \\
\hline $\mathrm{C} 7$ & $0.038(5)$ & $0.045(6)$ & $0.075(7)$ & -0.010 & $0.009(5)$ & $0.010(5)$ \\
\hline $\mathrm{C} 8$ & $0.082(7)$ & $0.051(6)$ & $0.037(5)$ & $-0.030(6)$ & $0.008(5)$ & $-0.015(5)$ \\
\hline C9 & $0.052(6)$ & $0.030(5)$ & $0.097(9)$ & $-0.002(5)$ & $-0.014(6)$ & $0.000(5)$ \\
\hline N5 & $0.062(6)$ & $0.027(5)$ & $0.111(9)$ & 0.005 (4) & $-0.012(5)$ & $-0.007(5)$ \\
\hline $\mathrm{C} 4$ & $0.051(5)$ & $0.051(6)$ & $0.036(5)$ & $0.013(5)$ & $-0.004(4)$ & -0.003 \\
\hline $\mathrm{C} 5$ & $0.055(6)$ & $0.062(7)$ & $0.050(6)$ & $0.026(5)$ & $0.018(5)$ & $-0.003(5)$ \\
\hline $\mathrm{C} 6$ & $0.058(6)$ & $0.033(5)$ & $0.089(9)$ & $0.001(5)$ & $0.005(6)$ & $0.001(5)$ \\
\hline $\mathrm{C} 12$ & $0.081(8)$ & $0.021(5)$ & $0.083(8)$ & $-0.016(5)$ & $-0.001(6)$ & $0.007(5)$ \\
\hline $\mathrm{C} 13$ & $0.060(6)$ & $0.040(5)$ & $0.030(5)$ & 0.019 (4) & $0.013(4)$ & -0.001 \\
\hline $\mathrm{C} 14$ & $0.040(5)$ & $0.037(5)$ & $0.037(5)$ & $0.001(4)$ & 0.008 & -0.001 \\
\hline $\mathrm{C} 15$ & $0.031(4)$ & $0.039(5)$ & $0.034(5)$ & $0.001(4)$ & $0.002(3)$ & -0.005 \\
\hline $\mathrm{C} 16$ & $0.055(6)$ & $0.046(6)$ & $0.048(6)$ & $0.016(5)$ & $0.019(4)$ & $0.022(5)$ \\
\hline
\end{tabular}




$\begin{array}{lllllll}\text { C17 } & 0.038(5) & 0.053(6) & 0.047(6) & 0.007(4) & -0.014(4) & 0.001(5) \\ \text { C18 } & 0.042(5) & 0.031(5) & 0.052(6) & 0.009(4) & 0.013(4) & 0.006(4) \\ \text { C19 } & 0.091(8) & 0.057(7) & 0.036(5) & -0.030(6) & 0.016(5) & -0.008(5) \\ \text { C20 } & 0.102(9) & 0.043(6) & 0.064(8) & 0.016(6) & -0.030(7) & 0.003(5) \\ \text { C21 } & 0.056(6) & 0.041(6) & 0.122(11) & -0.014(5) & 0.044(7) & -0.008(6) \\ \text { C22 } & 0.076(8) & 0.043(6) & 0.062(7) & -0.011(6) & -0.018(6) & 0.008(5) \\ \text { C23 } & 0.072(8) & 0.067(8) & 0.091(10) & -0.012(7) & 0.029(7) & -0.038(7) \\ \text { C24 } & 0.171(15) & 0.040(7) & 0.054(7) & -0.015(8) & 0.032(8) & 0.019(5) \\ \text { C25 } & 0.018(3) & 0.029(4) & 0.022(4) & -0.005(3) & 0.007(3) & 0.005(3) \\ \text { C26 } & 0.057(6) & 0.044(5) & 0.031(5) & -0.013(5) & -0.003(4) & -0.005(4) \\ \text { C27 } & 0.048(5) & 0.083(8) & 0.027(5) & 0.001(5) & 0.007(4) & -0.006(5) \\ \text { C28 } & 0.075(7) & 0.054(6) & 0.029(5) & -0.016(6) & 0.002(5) & 0.014(4) \\ \text { C29 } & 0.044(5) & 0.052(6) & 0.033(5) & 0.010(5) & -0.004(4) & 0.003(4) \\ \text { C30 } & 0.035(5) & 0.083(8) & 0.027(5) & -0.009(5) & -0.006(4) & 0.002(5) \\ \text { C31 } & 0.049(6) & 0.093(9) & 0.038(6) & -0.004(6) & -0.001(4) & -0.023(6) \\ \text { C32 } & 0.084(8) & 0.061(7) & 0.040(6) & -0.020(7) & 0.008(5) & -0.023(5) \\ \text { C33 } & 0.080(8) & 0.090(9) & 0.027(5) & 0.048(7) & -0.005(5) & -0.018(5) \\ \text { C34 } & 0.078(8) & 0.128(12) & 0.014(5) & -0.028(8) & 0.002(5) & 0.008(6) \\ \text { C35 } & 0.076(8) & 0.069(8) & 0.033(5) & 0.011(6) & -0.022(5) & -0.010(5) \\ \text { O1M } & 0.056(4) & 0.038(4) & 0.066(5) & -0.001(3) & 0.004(3) & -0.006(3) \\ \text { C1EB } & 0.067(7) & 0.067(7) & 0.093(9) & -0.012(6) & 0.017(6) & -0.002(6) \\ \text { C2E } & 0.067(7) & 0.067(7) & 0.093(9) & -0.012(6) & 0.017(6) & -0.002(6) \\ \text { C1EA } & 0.067(7) & 0.067(7) & 0.093(9) & -0.012(6) & 0.017(6) & -0.002(6)\end{array}$

Geometric parameters $(\AA, \stackrel{\circ}{)})$

\begin{tabular}{|c|c|c|c|}
\hline Re1-N101 & $1.720(10)$ & $\mathrm{N} 3-\mathrm{C} 2$ & $1.476(11)$ \\
\hline $\mathrm{Re} 1-\mathrm{O} 1 \mathrm{M}$ & $2.147(6)$ & $\mathrm{N} 3-\mathrm{C} 4$ & $1.491(12)$ \\
\hline $\mathrm{Re} 1-\mathrm{Br} 2$ & $2.5085(10)$ & $\mathrm{N} 4-\mathrm{C} 10$ & $1.435(15)$ \\
\hline $\mathrm{Re} 1-\mathrm{Br} 4$ & $2.5200(10)$ & $\mathrm{N} 4-\mathrm{C} 7$ & $1.465(13)$ \\
\hline $\operatorname{Re} 1-\mathrm{Br} 1$ & $2.5206(10)$ & $\mathrm{N} 4-\mathrm{C} 12$ & $1.466(12)$ \\
\hline $\operatorname{Re} 1-\mathrm{Br} 3$ & $2.5245(10)$ & $\mathrm{N} 6-\mathrm{C} 10$ & $1.475(16)$ \\
\hline $\mathrm{Ru} 1-\mathrm{C} 25$ & $2.008(7)$ & N6-C11 & $1.478(17)$ \\
\hline $\mathrm{Ru} 1-\mathrm{C} 28$ & $2.212(9)$ & $\mathrm{N} 6-\mathrm{C} 8$ & $1.479(12)$ \\
\hline $\mathrm{Ru} 1-\mathrm{C} 27$ & $2.214(9)$ & $\mathrm{N} 7-\mathrm{C} 16$ & $1.437(12)$ \\
\hline $\mathrm{Ru} 1-\mathrm{C} 29$ & $2.235(8)$ & $\mathrm{N} 7-\mathrm{C} 17$ & $1.457(12)$ \\
\hline $\mathrm{Ru} 1-\mathrm{P} 2$ & $2.243(2)$ & $\mathrm{N} 7-\mathrm{C} 13$ & $1.491(11)$ \\
\hline $\mathrm{Ru} 1-\mathrm{C} 30$ & $2.258(8)$ & $\mathrm{N} 8-\mathrm{C} 16$ & $1.475(11)$ \\
\hline $\mathrm{Ru} 1-\mathrm{C} 26$ & $2.261(8)$ & $\mathrm{N} 8-\mathrm{C} 18$ & $1.480(11)$ \\
\hline $\mathrm{Ru} 1-\mathrm{P} 1$ & $2.281(2)$ & $\mathrm{N} 8-\mathrm{C} 14$ & $1.482(10)$ \\
\hline $\mathrm{Ru} 2-\mathrm{N} 13$ & $2.030(8)$ & N9- $-\mathrm{C} 17$ & $1.454(12)$ \\
\hline $\mathrm{Ru} 2-\mathrm{C} 33$ & $2.198(9)$ & N9- $-\mathrm{C} 18$ & $1.454(11)$ \\
\hline $\mathrm{Ru} 2-\mathrm{C} 34$ & $2.202(9)$ & N9-C15 & $1.458(11)$ \\
\hline $\mathrm{Ru} 2-\mathrm{C} 32$ & $2.229(9)$ & $\mathrm{N} 10-\mathrm{C} 23$ & $1.437(16)$ \\
\hline $\mathrm{Ru} 2-\mathrm{C} 35$ & $2.245(9)$ & $\mathrm{N} 10-\mathrm{C} 22$ & $1.440(14)$ \\
\hline $\mathrm{Ru} 2-\mathrm{C} 31$ & $2.255(9)$ & $\mathrm{N} 10-\mathrm{C} 19$ & $1.476(13)$ \\
\hline $\mathrm{Ru} 2-\mathrm{P} 3$ & $2.268(2)$ & $\mathrm{N} 11-\mathrm{C} 23$ & $1.446(16)$ \\
\hline $\mathrm{Ru} 2-\mathrm{P} 4$ & $2.277(2)$ & $\mathrm{N} 11-\mathrm{C} 24$ & $1.453(17)$ \\
\hline
\end{tabular}




\begin{tabular}{|c|c|c|c|}
\hline $\mathrm{P} 1-\mathrm{C} 1$ & $1.840(8)$ & $\mathrm{N} 11-\mathrm{C} 20$ & $1.482(14)$ \\
\hline $\mathrm{P} 1-\mathrm{C} 3$ & $1.846(9)$ & $\mathrm{N} 12-\mathrm{C} 22$ & $1.433(14)$ \\
\hline $\mathrm{P} 1-\mathrm{C} 2$ & $1.850(8)$ & $\mathrm{N} 12-\mathrm{C} 24$ & $1.462(16)$ \\
\hline $\mathrm{P} 2-\mathrm{C} 7$ & $1.827(9)$ & $\mathrm{N} 12-\mathrm{C} 21$ & $1.498(13)$ \\
\hline $\mathrm{P} 2-\mathrm{C} 8$ & $1.838(9)$ & $\mathrm{C} 11-\mathrm{N} 5$ & $1.444(17)$ \\
\hline $\mathrm{P} 2-\mathrm{C} 9$ & $1.846(10)$ & $\mathrm{N} 13-\mathrm{C} 25$ & $1.142(10)$ \\
\hline $\mathrm{P} 3-\mathrm{C} 13$ & $1.841(8)$ & $\mathrm{C} 9-\mathrm{N} 5$ & $1.473(13)$ \\
\hline $\mathrm{P} 3-\mathrm{C} 15$ & $1.843(8)$ & $\mathrm{N} 5-\mathrm{C} 12$ & $1.464(14)$ \\
\hline $\mathrm{P} 3-\mathrm{C} 14$ & $1.853(9)$ & $\mathrm{C} 26-\mathrm{C} 30$ & $1.388(14)$ \\
\hline $\mathrm{P} 4-\mathrm{C} 20$ & $1.831(10)$ & $\mathrm{C} 26-\mathrm{C} 27$ & $1.441(14)$ \\
\hline $\mathrm{P} 4-\mathrm{C} 21$ & $1.834(10)$ & $\mathrm{C} 27-\mathrm{C} 28$ & $1.410(15)$ \\
\hline $\mathrm{P} 4-\mathrm{C} 19$ & $1.847(9)$ & $\mathrm{C} 28-\mathrm{C} 29$ & $1.417(14)$ \\
\hline $\mathrm{O} 101-\mathrm{N} 101$ & $1.183(11)$ & $\mathrm{C} 29-\mathrm{C} 30$ & $1.409(14)$ \\
\hline $\mathrm{N} 1-\mathrm{C} 5$ & 1.447 (12) & $\mathrm{C} 31-\mathrm{C} 32$ & $1.357(16)$ \\
\hline $\mathrm{N} 1-\mathrm{C} 1$ & $1.456(11)$ & $\mathrm{C} 31-\mathrm{C} 35$ & $1.411(16)$ \\
\hline $\mathrm{N} 1-\mathrm{C} 4$ & $1.458(12)$ & $\mathrm{C} 32-\mathrm{C} 33$ & $1.387(16)$ \\
\hline $\mathrm{N} 2-\mathrm{C} 6$ & $1.434(14)$ & $\mathrm{C} 33-\mathrm{C} 34$ & $1.410(17)$ \\
\hline $\mathrm{N} 2-\mathrm{C} 3$ & $1.451(12)$ & $\mathrm{C} 34-\mathrm{C} 35$ & $1.456(16)$ \\
\hline $\mathrm{N} 2-\mathrm{C} 5$ & $1.470(13)$ & $\mathrm{O} 1 \mathrm{M}-\mathrm{C} 1 \mathrm{~EB}$ & $1.471(13)$ \\
\hline $\mathrm{N} 3-\mathrm{C} 6$ & $1.473(13)$ & $\mathrm{C} 1 \mathrm{~EB}-\mathrm{C} 2 \mathrm{E}$ & $1.43(3)$ \\
\hline $\mathrm{N} 101-\mathrm{Re} 1-\mathrm{O} 1 \mathrm{M}$ & $178.9(3)$ & $\mathrm{C} 5-\mathrm{N} 1-\mathrm{C} 1$ & $112.1(7)$ \\
\hline N101-Re1-Br2 & $94.1(3)$ & $\mathrm{C} 5-\mathrm{N} 1-\mathrm{C} 4$ & $108.7(8)$ \\
\hline $\mathrm{O} 1 \mathrm{M}-\mathrm{Re} 1-\mathrm{Br} 2$ & 85.44 (19) & $\mathrm{C} 1-\mathrm{N} 1-\mathrm{C} 4$ & $111.3(7)$ \\
\hline N101-Re1-Br4 & $94.1(3)$ & $\mathrm{C} 6-\mathrm{N} 2-\mathrm{C} 3$ & $111.5(8)$ \\
\hline $\mathrm{O} 1 \mathrm{M}-\mathrm{Re} 1-\mathrm{Br} 4$ & 86.33 (19) & $\mathrm{C} 6-\mathrm{N} 2-\mathrm{C} 5$ & $108.7(8)$ \\
\hline $\mathrm{Br} 2-\mathrm{Re} 1-\mathrm{Br} 4$ & $171.77(4)$ & $\mathrm{C} 3-\mathrm{N} 2-\mathrm{C} 5$ & $110.8(8)$ \\
\hline $\mathrm{N} 101-\operatorname{Re} 1-\mathrm{Br} 1$ & $93.0(3)$ & $\mathrm{C} 6-\mathrm{N} 3-\mathrm{C} 2$ & $110.6(8)$ \\
\hline $\mathrm{O} 1 \mathrm{M}-\mathrm{Re} 1-\mathrm{Br} 1$ & $85.96(18)$ & $\mathrm{C} 6-\mathrm{N} 3-\mathrm{C} 4$ & $107.7(8)$ \\
\hline $\mathrm{Br} 2-\mathrm{Re} 1-\mathrm{Br} 1$ & $89.58(4)$ & $\mathrm{C} 2-\mathrm{N} 3-\mathrm{C} 4$ & $110.6(7)$ \\
\hline $\mathrm{Br} 4-\mathrm{Re} 1-\mathrm{Br} 1$ & $90.10(4)$ & $\mathrm{C} 10-\mathrm{N} 4-\mathrm{C} 7$ & $112.1(8)$ \\
\hline N101-Re1-Br3 & $93.0(3)$ & $\mathrm{C} 10-\mathrm{N} 4-\mathrm{C} 12$ & $109.5(10)$ \\
\hline $\mathrm{O} 1 \mathrm{M}-\mathrm{Re} 1-\mathrm{Br} 3$ & $87.99(18)$ & $\mathrm{C} 7-\mathrm{N} 4-\mathrm{C} 12$ & $110.8(8)$ \\
\hline $\mathrm{Br} 2-\mathrm{Re} 1-\mathrm{Br} 3$ & $89.45(4)$ & $\mathrm{C} 10-\mathrm{N} 6-\mathrm{C} 11$ & $107.6(9)$ \\
\hline $\mathrm{Br} 4-\mathrm{Re} 1-\mathrm{Br} 3$ & 90.01 (4) & $\mathrm{C} 10-\mathrm{N} 6-\mathrm{C} 8$ & $111.2(9)$ \\
\hline $\mathrm{Br} 1-\operatorname{Re} 1-\mathrm{Br} 3$ & $173.93(4)$ & $\mathrm{C} 11-\mathrm{N} 6-\mathrm{C} 8$ & $110.6(9)$ \\
\hline $\mathrm{C} 25-\mathrm{Ru} 1-\mathrm{C} 28$ & $142.5(4)$ & $\mathrm{C} 16-\mathrm{N} 7-\mathrm{C} 17$ & $109.7(7)$ \\
\hline $\mathrm{C} 25-\mathrm{Ru} 1-\mathrm{C} 27$ & $154.2(3)$ & $\mathrm{C} 16-\mathrm{N} 7-\mathrm{C} 13$ & $112.1(7)$ \\
\hline $\mathrm{C} 28-\mathrm{Ru} 1-\mathrm{C} 27$ & $37.2(4)$ & $\mathrm{C} 17-\mathrm{N} 7-\mathrm{C} 13$ & $109.3(7)$ \\
\hline $\mathrm{C} 25-\mathrm{Ru} 1-\mathrm{C} 29$ & $106.9(3)$ & $\mathrm{C} 16-\mathrm{N} 8-\mathrm{C} 18$ & $107.6(7)$ \\
\hline $\mathrm{C} 28-\mathrm{Ru} 1-\mathrm{C} 29$ & $37.1(4)$ & $\mathrm{C} 16-\mathrm{N} 8-\mathrm{C} 14$ & $110.3(6)$ \\
\hline $\mathrm{C} 27-\mathrm{Ru} 1-\mathrm{C} 29$ & $61.3(4)$ & $\mathrm{C} 18-\mathrm{N} 8-\mathrm{C} 14$ & $110.3(7)$ \\
\hline $\mathrm{C} 25-\mathrm{Ru} 1-\mathrm{P} 2$ & $86.3(2)$ & $\mathrm{C} 17-\mathrm{N} 9-\mathrm{C} 18$ & $108.8(7)$ \\
\hline $\mathrm{C} 28-\mathrm{Ru} 1-\mathrm{P} 2$ & $91.3(3)$ & $\mathrm{C} 17-\mathrm{N} 9-\mathrm{C} 15$ & $110.8(7)$ \\
\hline $\mathrm{C} 27-\mathrm{Ru} 1-\mathrm{P} 2$ & $117.6(3)$ & $\mathrm{C} 18-\mathrm{N} 9-\mathrm{C} 15$ & $113.2(7)$ \\
\hline $\mathrm{C} 29-\mathrm{Ru} 1-\mathrm{P} 2$ & $101.4(3)$ & $\mathrm{C} 23-\mathrm{N} 10-\mathrm{C} 22$ & $109.8(9)$ \\
\hline $\mathrm{C} 25-\mathrm{Ru} 1-\mathrm{C} 30$ & $95.6(3)$ & $\mathrm{C} 23-\mathrm{N} 10-\mathrm{C} 19$ & $110.3(9)$ \\
\hline $\mathrm{C} 28-\mathrm{Ru} 1-\mathrm{C} 30$ & $61.6(4)$ & $\mathrm{C} 22-\mathrm{N} 10-\mathrm{C} 19$ & $110.3(9)$ \\
\hline
\end{tabular}




\begin{tabular}{|c|c|c|c|}
\hline $\mathrm{C} 27-\mathrm{Ru} 1-\mathrm{C} 30$ & $60.9(3)$ & $\mathrm{C} 23-\mathrm{N} 11-\mathrm{C} 24$ & $110.5(10)$ \\
\hline $\mathrm{C} 29-\mathrm{Ru} 1-\mathrm{C} 30$ & $36.5(4)$ & $\mathrm{C} 23-\mathrm{N} 11-\mathrm{C} 20$ & $111.6(10)$ \\
\hline $\mathrm{P} 2-\mathrm{Ru} 1-\mathrm{C} 30$ & $136.4(3)$ & $\mathrm{C} 24-\mathrm{N} 11-\mathrm{C} 20$ & $107.9(11)$ \\
\hline $\mathrm{C} 25-\mathrm{Ru} 1-\mathrm{C} 26$ & $117.0(3)$ & $\mathrm{C} 22-\mathrm{N} 12-\mathrm{C} 24$ & $109.1(9)$ \\
\hline $\mathrm{C} 28-\mathrm{Ru} 1-\mathrm{C} 26$ & $62.5(4)$ & $\mathrm{C} 22-\mathrm{N} 12-\mathrm{C} 21$ & $110.1(9)$ \\
\hline $\mathrm{C} 27-\mathrm{Ru} 1-\mathrm{C} 26$ & $37.5(4)$ & $\mathrm{C} 24-\mathrm{N} 12-\mathrm{C} 21$ & $109.4(10)$ \\
\hline $\mathrm{C} 29-\mathrm{Ru} 1-\mathrm{C} 26$ & $61.1(4)$ & $\mathrm{N} 4-\mathrm{C} 10-\mathrm{N} 6$ & $113.8(8)$ \\
\hline $\mathrm{P} 2-\mathrm{Ru} 1-\mathrm{C} 26$ & $153.3(2)$ & $\mathrm{N} 5-\mathrm{C} 11-\mathrm{N} 6$ & $116.1(10)$ \\
\hline $\mathrm{C} 30-\mathrm{Ru} 1-\mathrm{C} 26$ & $35.8(3)$ & $\mathrm{C} 25-\mathrm{N} 13-\mathrm{Ru} 2$ & $176.3(7)$ \\
\hline $\mathrm{C} 25-\mathrm{Ru} 1-\mathrm{P} 1$ & $88.1(2)$ & $\mathrm{O} 101-\mathrm{N} 101-\mathrm{Re} 1$ & $178.6(10)$ \\
\hline $\mathrm{C} 28-\mathrm{Ru} 1-\mathrm{P} 1$ & $129.4(3)$ & $\mathrm{N} 1-\mathrm{C} 1-\mathrm{P} 1$ & $113.5(6)$ \\
\hline $\mathrm{C} 27-\mathrm{Ru} 1-\mathrm{P} 1$ & $98.1(3)$ & $\mathrm{N} 3-\mathrm{C} 2-\mathrm{P} 1$ & $113.1(6)$ \\
\hline $\mathrm{C} 29-\mathrm{Ru} 1-\mathrm{P} 1$ & $157.7(3)$ & $\mathrm{N} 2-\mathrm{C} 3-\mathrm{P} 1$ & $113.4(6)$ \\
\hline P2-Ru1-P1 & $95.97(8)$ & $\mathrm{N} 4-\mathrm{C} 7-\mathrm{P} 2$ & $112.5(7)$ \\
\hline $\mathrm{C} 30-\mathrm{Ru} 1-\mathrm{P} 1$ & $127.6(3)$ & $\mathrm{N} 6-\mathrm{C} 8-\mathrm{P} 2$ & $111.6(7)$ \\
\hline $\mathrm{C} 26-\mathrm{Ru} 1-\mathrm{P} 1$ & $97.5(3)$ & $\mathrm{N} 5-\mathrm{C} 9-\mathrm{P} 2$ & $112.7(7)$ \\
\hline N13-Ru2-C33 & $145.0(4)$ & $\mathrm{C} 11-\mathrm{N} 5-\mathrm{C} 12$ & $106.9(9)$ \\
\hline $\mathrm{N} 13-\mathrm{Ru} 2-\mathrm{C} 34$ & $151.6(4)$ & $\mathrm{C} 11-\mathrm{N} 5-\mathrm{C} 9$ & $111.3(10)$ \\
\hline $\mathrm{C} 33-\mathrm{Ru} 2-\mathrm{C} 34$ & $37.4(5)$ & $\mathrm{C} 12-\mathrm{N} 5-\mathrm{C} 9$ & $110.9(9)$ \\
\hline $\mathrm{N} 13-\mathrm{Ru} 2-\mathrm{C} 32$ & $109.3(4)$ & $\mathrm{N} 1-\mathrm{C} 4-\mathrm{N} 3$ & $113.3(7)$ \\
\hline $\mathrm{C} 33-\mathrm{Ru} 2-\mathrm{C} 32$ & $36.5(4)$ & $\mathrm{N} 1-\mathrm{C} 5-\mathrm{N} 2$ & $113.8(7)$ \\
\hline $\mathrm{C} 34-\mathrm{Ru} 2-\mathrm{C} 32$ & $60.9(4)$ & $\mathrm{N} 2-\mathrm{C} 6-\mathrm{N} 3$ & $115.1(8)$ \\
\hline N13-Ru2-C35 & $113.3(4)$ & $\mathrm{N} 5-\mathrm{C} 12-\mathrm{N} 4$ & $114.0(8)$ \\
\hline $\mathrm{C} 33-\mathrm{Ru} 2-\mathrm{C} 35$ & $62.8(4)$ & $\mathrm{N} 7-\mathrm{C} 13-\mathrm{P} 3$ & $112.5(5)$ \\
\hline C34-Ru2-C35 & $38.2(4)$ & $\mathrm{N} 8-\mathrm{C} 14-\mathrm{P} 3$ & $114.2(6)$ \\
\hline $\mathrm{C} 32-\mathrm{Ru} 2-\mathrm{C} 35$ & $60.6(4)$ & $\mathrm{N} 9-\mathrm{C} 15-\mathrm{P} 3$ & $112.3(6)$ \\
\hline N13-Ru2-C31 & $94.7(3)$ & $\mathrm{N} 7-\mathrm{C} 16-\mathrm{N} 8$ & $114.5(7)$ \\
\hline $\mathrm{C} 33-\mathrm{Ru} 2-\mathrm{C} 31$ & $60.9(4)$ & $\mathrm{N} 9-\mathrm{C} 17-\mathrm{N} 7$ & $114.3(7)$ \\
\hline $\mathrm{C} 34-\mathrm{Ru} 2-\mathrm{C} 31$ & $61.5(4)$ & $\mathrm{N} 9-\mathrm{C} 18-\mathrm{N} 8$ & $113.6(7)$ \\
\hline $\mathrm{C} 32-\mathrm{Ru} 2-\mathrm{C} 31$ & $35.2(4)$ & $\mathrm{N} 10-\mathrm{C} 19-\mathrm{P} 4$ & $112.9(7)$ \\
\hline $\mathrm{C} 35-\mathrm{Ru} 2-\mathrm{C} 31$ & $36.6(4)$ & $\mathrm{N} 11-\mathrm{C} 20-\mathrm{P} 4$ & $113.0(7)$ \\
\hline $\mathrm{N} 13-\mathrm{Ru} 2-\mathrm{P} 3$ & $86.25(19)$ & $\mathrm{N} 12-\mathrm{C} 21-\mathrm{P} 4$ & $112.5(7)$ \\
\hline $\mathrm{C} 33-\mathrm{Ru} 2-\mathrm{P} 3$ & $94.2(3)$ & $\mathrm{N} 12-\mathrm{C} 22-\mathrm{N} 10$ & $115.9(9)$ \\
\hline $\mathrm{C} 34-\mathrm{Ru} 2-\mathrm{P} 3$ & $121.3(4)$ & $\mathrm{N} 10-\mathrm{C} 23-\mathrm{N} 11$ & $114.2(9)$ \\
\hline $\mathrm{C} 32-\mathrm{Ru} 2-\mathrm{P} 3$ & $102.5(3)$ & $\mathrm{N} 11-\mathrm{C} 24-\mathrm{N} 12$ & $114.9(9)$ \\
\hline $\mathrm{C} 35-\mathrm{Ru} 2-\mathrm{P} 3$ & $156.9(3)$ & N13-C25-Ru1 & $175.5(7)$ \\
\hline $\mathrm{C} 31-\mathrm{Ru} 2-\mathrm{P} 3$ & $134.9(3)$ & $\mathrm{C} 30-\mathrm{C} 26-\mathrm{C} 27$ & $106.4(9)$ \\
\hline $\mathrm{N} 13-\mathrm{Ru} 2-\mathrm{P} 4$ & $85.8(2)$ & $\mathrm{C} 30-\mathrm{C} 26-\mathrm{Ru} 1$ & $72.0(5)$ \\
\hline $\mathrm{C} 33-\mathrm{Ru} 2-\mathrm{P} 4$ & $128.6(4)$ & $\mathrm{C} 27-\mathrm{C} 26-\mathrm{Ru} 1$ & $69.4(5)$ \\
\hline $\mathrm{C} 34-\mathrm{Ru} 2-\mathrm{P} 4$ & $96.7(4)$ & $\mathrm{C} 28-\mathrm{C} 27-\mathrm{C} 26$ & $108.9(9)$ \\
\hline $\mathrm{C} 32-\mathrm{Ru} 2-\mathrm{P} 4$ & $155.8(3)$ & $\mathrm{C} 28-\mathrm{C} 27-\mathrm{Ru} 1$ & $71.4(5)$ \\
\hline $\mathrm{C} 35-\mathrm{Ru} 2-\mathrm{P} 4$ & $96.4(3)$ & $\mathrm{C} 26-\mathrm{C} 27-\mathrm{Ru} 1$ & $73.0(5)$ \\
\hline $\mathrm{C} 31-\mathrm{Ru} 2-\mathrm{P} 4$ & $127.9(3)$ & $\mathrm{C} 27-\mathrm{C} 28-\mathrm{C} 29$ & $106.7(9)$ \\
\hline $\mathrm{P} 3-\mathrm{Ru} 2-\mathrm{P} 4$ & $97.13(7)$ & $\mathrm{C} 27-\mathrm{C} 28-\mathrm{Ru} 1$ & $71.5(5)$ \\
\hline $\mathrm{C} 1-\mathrm{P} 1-\mathrm{C} 3$ & $96.6(5)$ & $\mathrm{C} 29-\mathrm{C} 28-\mathrm{Ru} 1$ & $72.3(5)$ \\
\hline $\mathrm{C} 1-\mathrm{P} 1-\mathrm{C} 2$ & $96.5(4)$ & $\mathrm{C} 30-\mathrm{C} 29-\mathrm{C} 28$ & $108.3(9)$ \\
\hline $\mathrm{C} 3-\mathrm{P} 1-\mathrm{C} 2$ & $97.4(5)$ & $\mathrm{C} 30-\mathrm{C} 29-\mathrm{Ru} 1$ & $72.6(5)$ \\
\hline $\mathrm{C} 1-\mathrm{P} 1-\mathrm{Ru} 1$ & $126.3(3)$ & $\mathrm{C} 28-\mathrm{C} 29-\mathrm{Ru} 1$ & $70.6(5)$ \\
\hline
\end{tabular}




\begin{tabular}{|c|c|}
\hline $\mathrm{C} 3-\mathrm{P} 1-\mathrm{Ru} 1$ & $114.4(3)$ \\
\hline $\mathrm{C} 2-\mathrm{P} 1-\mathrm{Ru} 1$ & $119.8(3)$ \\
\hline $\mathrm{C} 7-\mathrm{P} 2-\mathrm{C} 8$ & $99.0(5)$ \\
\hline $\mathrm{C} 7-\mathrm{P} 2-\mathrm{C} 9$ & $96.6(5)$ \\
\hline $\mathrm{C} 8-\mathrm{P} 2-\mathrm{C} 9$ & $98.5(6)$ \\
\hline $\mathrm{C} 7-\mathrm{P} 2-\mathrm{Ru} 1$ & $122.8(4)$ \\
\hline $\mathrm{C} 8-\mathrm{P} 2-\mathrm{Ru} 1$ & $120.6(3)$ \\
\hline $\mathrm{C} 9-\mathrm{P} 2-\mathrm{Ru} 1$ & $114.4(3)$ \\
\hline $\mathrm{C} 13-\mathrm{P} 3-\mathrm{C} 15$ & $97.8(4)$ \\
\hline $\mathrm{C} 13-\mathrm{P} 3-\mathrm{C} 14$ & $96.6(4)$ \\
\hline $\mathrm{C} 15-\mathrm{P} 3-\mathrm{C} 14$ & $96.9(4)$ \\
\hline $\mathrm{C} 13-\mathrm{P} 3-\mathrm{Ru} 2$ & $120.6(3)$ \\
\hline $\mathrm{C} 15-\mathrm{P} 3-\mathrm{Ru} 2$ & $124.4(3)$ \\
\hline C14-P3-Ru2 & $115.0(3)$ \\
\hline $\mathrm{C} 20-\mathrm{P} 4-\mathrm{C} 21$ & $97.7(6)$ \\
\hline $\mathrm{C} 20-\mathrm{P} 4-\mathrm{C} 19$ & $97.3(6)$ \\
\hline $\mathrm{C} 21-\mathrm{P} 4-\mathrm{C} 19$ & $96.7(5)$ \\
\hline $\mathrm{C} 20-\mathrm{P} 4-\mathrm{Ru} 2$ & $113.5(4)$ \\
\hline $\mathrm{C} 21-\mathrm{P} 4-\mathrm{Ru} 2$ & $125.0(4)$ \\
\hline $\mathrm{C} 19-\mathrm{P} 4-\mathrm{Ru} 2$ & $121.1(3)$ \\
\hline $\mathrm{C} 7-\mathrm{N} 4-\mathrm{C} 10-\mathrm{N} 6$ & $67.9(11)$ \\
\hline $\mathrm{C} 12-\mathrm{N} 4-\mathrm{C} 10-\mathrm{N} 6$ & $-55.4(11)$ \\
\hline $\mathrm{C} 11-\mathrm{N} 6-\mathrm{C} 10-\mathrm{N} 4$ & $53.3(12)$ \\
\hline $\mathrm{C} 8-\mathrm{N} 6-\mathrm{C} 10-\mathrm{N} 4$ & $-68.0(12)$ \\
\hline $\mathrm{C} 10-\mathrm{N} 6-\mathrm{C} 11-\mathrm{N} 5$ & $-54.7(12)$ \\
\hline $\mathrm{C} 8-\mathrm{N} 6-\mathrm{C} 11-\mathrm{N} 5$ & $67.0(13)$ \\
\hline $\mathrm{C} 5-\mathrm{N} 1-\mathrm{C} 1-\mathrm{P} 1$ & $-59.7(9)$ \\
\hline $\mathrm{C} 4-\mathrm{N} 1-\mathrm{C} 1-\mathrm{P} 1$ & $62.2(9)$ \\
\hline $\mathrm{C} 3-\mathrm{P} 1-\mathrm{C} 1-\mathrm{N} 1$ & $48.0(7)$ \\
\hline $\mathrm{C} 2-\mathrm{P} 1-\mathrm{C} 1-\mathrm{N} 1$ & $-50.2(7)$ \\
\hline $\mathrm{Ru} 1-\mathrm{P} 1-\mathrm{C} 1-\mathrm{N} 1$ & $175.0(5)$ \\
\hline $\mathrm{C} 6-\mathrm{N} 3-\mathrm{C} 2-\mathrm{P} 1$ & $58.7(9)$ \\
\hline $\mathrm{C} 4-\mathrm{N} 3-\mathrm{C} 2-\mathrm{P} 1$ & $-60.6(9)$ \\
\hline $\mathrm{C} 1-\mathrm{P} 1-\mathrm{C} 2-\mathrm{N} 3$ & $49.7(8)$ \\
\hline $\mathrm{C} 3-\mathrm{P} 1-\mathrm{C} 2-\mathrm{N} 3$ & $-47.8(8)$ \\
\hline $\mathrm{Ru} 1-\mathrm{P} 1-\mathrm{C} 2-\mathrm{N} 3$ & $-171.5(5)$ \\
\hline $\mathrm{C} 6-\mathrm{N} 2-\mathrm{C} 3-\mathrm{P} 1$ & $-60.1(10)$ \\
\hline $\mathrm{C} 5-\mathrm{N} 2-\mathrm{C} 3-\mathrm{P} 1$ & $61.1(10)$ \\
\hline $\mathrm{C} 1-\mathrm{P} 1-\mathrm{C} 3-\mathrm{N} 2$ & $-49.3(8)$ \\
\hline $\mathrm{C} 2-\mathrm{P} 1-\mathrm{C} 3-\mathrm{N} 2$ & $48.1(8)$ \\
\hline $\mathrm{Ru} 1-\mathrm{P} 1-\mathrm{C} 3-\mathrm{N} 2$ & $175.7(6)$ \\
\hline $\mathrm{C} 10-\mathrm{N} 4-\mathrm{C} 7-\mathrm{P} 2$ & $-59.8(10)$ \\
\hline $\mathrm{C} 12-\mathrm{N} 4-\mathrm{C} 7-\mathrm{P} 2$ & $62.8(10)$ \\
\hline $\mathrm{C} 8-\mathrm{P} 2-\mathrm{C} 7-\mathrm{N} 4$ & $47.8(8)$ \\
\hline $\mathrm{C} 9-\mathrm{P} 2-\mathrm{C} 7-\mathrm{N} 4$ & $-52.0(8)$ \\
\hline $\mathrm{Ru} 1-\mathrm{P} 2-\mathrm{C} 7-\mathrm{N} 4$ & $-176.6(5)$ \\
\hline
\end{tabular}

$\begin{array}{ll}\mathrm{C} 26-\mathrm{C} 30-\mathrm{C} 29 & 109.6(8) \\ \mathrm{C} 26-\mathrm{C} 30-\mathrm{Ru} 1 & 72.2(5) \\ \mathrm{C} 29-\mathrm{C} 30-\mathrm{Ru} 1 & 70.8(5) \\ \mathrm{C} 32-\mathrm{C} 31-\mathrm{C} 35 & 109.4(10) \\ \mathrm{C} 32-\mathrm{C} 31-\mathrm{Ru} 2 & 71.4(6) \\ \mathrm{C} 35-\mathrm{C} 31-\mathrm{Ru} 2 & 71.4(5) \\ \mathrm{C} 31-\mathrm{C} 32-\mathrm{C} 33 & 110.7(11) \\ \mathrm{C} 31-\mathrm{C} 32-\mathrm{Ru} 2 & 73.4(6) \\ \mathrm{C} 33-\mathrm{C} 32-\mathrm{Ru} 2 & 70.5(6) \\ \mathrm{C} 32-\mathrm{C} 33-\mathrm{C} 34 & 106.9(10) \\ \mathrm{C} 32-\mathrm{C} 33-\mathrm{Ru} 2 & 73.0(6) \\ \mathrm{C} 34-\mathrm{C} 33-\mathrm{Ru} 2 & 71.4(6) \\ \mathrm{C} 33-\mathrm{C} 34-\mathrm{C} 35 & 107.8(11) \\ \mathrm{C} 33-\mathrm{C} 34-\mathrm{Ru} 2 & 71.2(6) \\ \mathrm{C} 35-\mathrm{C} 34-\mathrm{Ru} 2 & 72.5(6) \\ \mathrm{C} 31-\mathrm{C} 35-\mathrm{C} 34 & 105.2(10) \\ \mathrm{C} 31-\mathrm{C} 35-\mathrm{Ru} 2 & 72.1(6) \\ \mathrm{C} 34-\mathrm{C} 35-\mathrm{Ru} 2 & 69.3(5) \\ \mathrm{C} 1 \mathrm{~EB}-\mathrm{O} 1 \mathrm{M}-\mathrm{Re} 1 & 128.3(6) \\ \mathrm{C} 2 \mathrm{E}-\mathrm{C} 1 \mathrm{~EB}-\mathrm{O} 1 \mathrm{M} & 147.8(14)\end{array}$

$67.5(10)$

$54.6(9)$

$-65.7(10)$

$-54.8(10)$

$70.3(10)$

$53.9(10)$

$-69.3(10)$

$56.1(9)$

$-67.6(9)$

$-55.5(9)$

$64.8(9)$

$61.3(11)$

$-60.2(11)$

$-48.6(10)$

$50.1(10)$

$-171.8(7)$

$-59.0(14)$

$62.6(12)$

$-50.6(11)$

$47.2(11)$

$175.8(9)$

$60.6(12)$

$-59.3(12)$

$48.4(10)$

$-49.9(10)$

$174.3(7)$

$52.2(12)$ 


$\begin{array}{ll}\text { C11-N6-C8-P2 } & -60.0(12) \\ \text { C7-P2-C8-N6 } & -48.0(9) \\ \text { C9-P2-C8-N6 } & 50.1(9) \\ \text { Ru1-P2-C8-N6 } & 175.1(7) \\ \text { C7-P2-C9-N5 } & 50.9(10) \\ \text { C8-P2-C9-N5 } & -49.2(10) \\ \text { Ru1-P2-C9-N5 } & -178.5(8) \\ \text { N6-C11-N5-C12 } & 55.5(12) \\ \text { N6-C11-N5-C9 } & -65.7(13) \\ \text { P2-C9-N5-C11 } & 58.3(12) \\ \text { P2-C9-N5-C12 } & -60.5(12) \\ \text { C5-N1-C4-N3 } & 56.5(10) \\ \text { C1-N1-C4-N3 } & -67.4(10) \\ \text { C6-N3-C4-N1 } & -54.3(10) \\ \text { C2-N3-C4-N1 } & 66.7(10) \\ \text { C1-N1-C5-N2 } & 66.4(10) \\ \text { C4-N1-C5-N2 } & -57.0(10) \\ \text { C6-N2-C5-N1 } & 55.9(11) \\ \text { C3-N2-C5-N1 } & -67.0(10) \\ \text { C3-N2-C6-N3 } & 67.6(11) \\ \text { C5-N2-C6-N3 } & -54.8(11) \\ \text { C2-N3-C6-N2 } & -66.8(11) \\ \text { C4-N3-C6-N2 } & 54.2(11) \\ \text { C11-N5-C12-N4 } & -55.5(12) \\ \text { C9-N5-C12-N4 } & 66.0(13) \\ \text { C10-N4-C12-N5 } & 56.9(11) \\ \text { C7-N4-C12-N5 } & -67.2(12) \\ \text { C16-N7-C13-P3 } & -61.3(9) \\ \text { C17-N7-C13-P3 } & 60.6(9) \\ \text { C15-P3-C13-N7 } & -49.0(7) \\ \text { C14-P3-C13-N7 } & 48.9(7) \\ \text { Ru2-P3-C13-N7 } & 173.0(5) \\ \text { C16-N8-C14-P3 } & 59.5(8) \\ \text { C18-N8-C14-P3 } & -59.3(8) \\ \text { C13-P3-C14-N8 } & -49.6(7) \\ \text { C15-P3-C14-N8 } & 49.1(6) \\ \text { Ru2-P3-C14-N8 } & -177.7(5) \\ \text { C17-N9-C15-P3 } & -61.0(8) \\ \text { C18-N9-C15-P3 } & 61.6(8) \\ \text { C13-P3-C15-N9 } & 49.0(7) \\ \text { C14-P3-C15-N9 } & -48.7(6) \\ \text { Ru2-P3-C15-N9 } & -175.4(4) \\ \text { C17-N7-C16-N8 } & -54.2(10) \\ & \end{array}$

\begin{tabular}{|c|c|}
\hline $\mathrm{C} 21-\mathrm{N} 12-\mathrm{C} 22-\mathrm{N} 10$ & $-67.9(12)$ \\
\hline $\mathrm{C} 23-\mathrm{N} 10-\mathrm{C} 22-\mathrm{N} 12$ & $-53.9(12)$ \\
\hline $\mathrm{C} 19-\mathrm{N} 10-\mathrm{C} 22-\mathrm{N} 12$ & $67.9(12)$ \\
\hline $\mathrm{C} 22-\mathrm{N} 10-\mathrm{C} 23-\mathrm{N} 11$ & $52.8(12)$ \\
\hline $\mathrm{C} 19-\mathrm{N} 10-\mathrm{C} 23-\mathrm{N} 11$ & $-69.0(12)$ \\
\hline $\mathrm{C} 24-\mathrm{N} 11-\mathrm{C} 23-\mathrm{N} 10$ & $-52.0(13)$ \\
\hline $\mathrm{C} 20-\mathrm{N} 11-\mathrm{C} 23-\mathrm{N} 10$ & $68.0(13)$ \\
\hline $\mathrm{C} 23-\mathrm{N} 11-\mathrm{C} 24-\mathrm{N} 12$ & $50.9(14)$ \\
\hline $\mathrm{C} 20-\mathrm{N} 11-\mathrm{C} 24-\mathrm{N} 12$ & $-71.3(13)$ \\
\hline $\mathrm{C} 22-\mathrm{N} 12-\mathrm{C} 24-\mathrm{N} 11$ & $-50.4(13)$ \\
\hline $\mathrm{C} 21-\mathrm{N} 12-\mathrm{C} 24-\mathrm{N} 11$ & $70.2(13)$ \\
\hline $\mathrm{C} 30-\mathrm{C} 26-\mathrm{C} 27-\mathrm{C} 28$ & $-0.2(10)$ \\
\hline $\mathrm{Ru} 1-\mathrm{C} 26-\mathrm{C} 27-\mathrm{C} 28$ & $62.7(6)$ \\
\hline $\mathrm{C} 30-\mathrm{C} 26-\mathrm{C} 27-\mathrm{Ru} 1$ & $-63.0(6)$ \\
\hline $\mathrm{C} 26-\mathrm{C} 27-\mathrm{C} 28-\mathrm{C} 29$ & $0.4(10)$ \\
\hline $\mathrm{Ru} 1-\mathrm{C} 27-\mathrm{C} 28-\mathrm{C} 29$ & $64.2(6)$ \\
\hline $\mathrm{C} 26-\mathrm{C} 27-\mathrm{C} 28-\mathrm{Ru} 1$ & $-63.8(6)$ \\
\hline $\mathrm{C} 27-\mathrm{C} 28-\mathrm{C} 29-\mathrm{C} 30$ & $-0.4(10)$ \\
\hline $\mathrm{Ru} 1-\mathrm{C} 28-\mathrm{C} 29-\mathrm{C} 30$ & $63.2(6)$ \\
\hline $\mathrm{C} 27-\mathrm{C} 28-\mathrm{C} 29-\mathrm{Ru} 1$ & $-63.6(6)$ \\
\hline $\mathrm{C} 27-\mathrm{C} 26-\mathrm{C} 30-\mathrm{C} 29$ & $0.0(10)$ \\
\hline $\mathrm{Ru} 1-\mathrm{C} 26-\mathrm{C} 30-\mathrm{C} 29$ & $-61.3(6)$ \\
\hline $\mathrm{C} 27-\mathrm{C} 26-\mathrm{C} 30-\mathrm{Ru} 1$ & $61.3(6)$ \\
\hline $\mathrm{C} 28-\mathrm{C} 29-\mathrm{C} 30-\mathrm{C} 26$ & $0.3(10)$ \\
\hline $\mathrm{Ru} 1-\mathrm{C} 29-\mathrm{C} 30-\mathrm{C} 26$ & $62.2(6)$ \\
\hline $\mathrm{C} 28-\mathrm{C} 29-\mathrm{C} 30-\mathrm{Ru} 1$ & $-61.9(6)$ \\
\hline $\mathrm{C} 35-\mathrm{C} 31-\mathrm{C} 32-\mathrm{C} 33$ & $0.5(12)$ \\
\hline $\mathrm{Ru} 2-\mathrm{C} 31-\mathrm{C} 32-\mathrm{C} 33$ & $-61.0(7)$ \\
\hline $\mathrm{C} 35-\mathrm{C} 31-\mathrm{C} 32-\mathrm{Ru} 2$ & $61.6(7)$ \\
\hline $\mathrm{C} 31-\mathrm{C} 32-\mathrm{C} 33-\mathrm{C} 34$ & $-1.1(11)$ \\
\hline $\mathrm{Ru} 2-\mathrm{C} 32-\mathrm{C} 33-\mathrm{C} 34$ & $-63.8(7)$ \\
\hline $\mathrm{C} 31-\mathrm{C} 32-\mathrm{C} 33-\mathrm{Ru} 2$ & $62.8(7)$ \\
\hline $\mathrm{C} 32-\mathrm{C} 33-\mathrm{C} 34-\mathrm{C} 35$ & $1.2(11)$ \\
\hline $\mathrm{Ru} 2-\mathrm{C} 33-\mathrm{C} 34-\mathrm{C} 35$ & $-63.7(6)$ \\
\hline $\mathrm{C} 32-\mathrm{C} 33-\mathrm{C} 34-\mathrm{Ru} 2$ & $64.9(7)$ \\
\hline $\mathrm{C} 32-\mathrm{C} 31-\mathrm{C} 35-\mathrm{C} 34$ & $0.2(11)$ \\
\hline $\mathrm{Ru} 2-\mathrm{C} 31-\mathrm{C} 35-\mathrm{C} 34$ & $61.8(6)$ \\
\hline $\mathrm{C} 32-\mathrm{C} 31-\mathrm{C} 35-\mathrm{Ru} 2$ & $-61.6(7)$ \\
\hline $\mathrm{C} 33-\mathrm{C} 34-\mathrm{C} 35-\mathrm{C} 31$ & $-0.8(10)$ \\
\hline $\mathrm{Ru} 2-\mathrm{C} 34-\mathrm{C} 35-\mathrm{C} 31$ & $-63.7(7)$ \\
\hline $\mathrm{C} 33-\mathrm{C} 34-\mathrm{C} 35-\mathrm{Ru} 2$ & $62.8(6)$ \\
\hline $\mathrm{Re} 1-\mathrm{O} 1 \mathrm{M}-\mathrm{C} 1 \mathrm{~EB}-\mathrm{C} 2 \mathrm{E}$ & $-144(3)$ \\
\hline
\end{tabular}

Hydrogen-bond geometry $\left(\AA,{ }^{\circ}\right)$

\begin{tabular}{lllll}
\hline$D-\mathrm{H} \cdots A$ & $D-\mathrm{H}$ & $\mathrm{H} \cdots A$ & $D \cdots A$ & $D-\mathrm{H} \cdots A$ \\
\hline $\mathrm{C} 10-\mathrm{H} 10 A \cdots \mathrm{Br}^{\mathrm{i}}$ & 0.97 & 3.12 & $3.944(12)$ & 143
\end{tabular}


supporting information

$\begin{array}{lllll}\mathrm{C} 10-\mathrm{H} 10 B \cdots \mathrm{Br} 2 & 0.97 & 2.83 & 3.709(10) & 150 \\ \mathrm{C} 1-\mathrm{H} 1 B^{\cdots} \cdots \mathrm{Br} 4^{4 i} & 0.97 & 3.03 & 3.967(9) & 163 \\ \mathrm{C} 7-\mathrm{H} 7 B \cdots \mathrm{N} 9^{\text {iii }} & 0.97 & 2.59 & 3.309(11) & 131 \\ \mathrm{C} 8-\mathrm{H} 8 A \cdots \mathrm{Br}^{\mathrm{ii}} & 0.97 & 2.89 & 3.772(12) & 151 \\ \mathrm{C} 4-\mathrm{H} 4 B \cdots \mathrm{Br}^{\mathrm{i}} & 0.97 & 3.10 & 4.062(10) & 169 \\ \mathrm{C} 5-\mathrm{H} 5 A \cdots \mathrm{Br} 1^{\mathrm{ii}} & 0.97 & 3.10 & 3.918(10) & 143 \\ \mathrm{C} 18-\mathrm{H} 18 A \cdots \mathrm{N} 4^{\mathrm{iv}} & 0.97 & 2.53 & 3.208(11) & 127 \\ \mathrm{C} 18-\mathrm{H} 18 B \cdots \mathrm{Br} 2^{\mathrm{v}} & 0.97 & 2.92 & 3.858(9) & 163 \\ \mathrm{C} 19-\mathrm{H} 19 B \cdots \mathrm{Br} 1^{\mathrm{vi}} & 0.97 & 3.09 & 3.938(11) & 147 \\ \mathrm{C} 22-\mathrm{H} 22 B \cdots \mathrm{Br} 1^{\mathrm{vi}} & 0.97 & 3.00 & 3.861(10) & 148 \\ \mathrm{C} 23-\mathrm{H} 23 A \cdots \mathrm{Br} 4^{\mathrm{vii}} & 0.97 & 3.10 & 4.007(12) & 156 \\ \mathrm{C} 24-\mathrm{H} 24 A \cdots \mathrm{Br} 3^{\mathrm{vii}} & 0.97 & 2.98 & 3.799(11) & 143 \\ \mathrm{O} 1 M-\mathrm{H} 1 m \cdots \mathrm{N} 8^{\mathrm{iii}} & 0.85 & 1.88 & 2.709(9) & 166 \\ \mathrm{C} 1 E B-\mathrm{H} 101 \cdots \mathrm{Br} 3 & 0.97 & 2.80 & 3.527(13) & 132 \\ \mathrm{C} 2 E-\mathrm{H} 2 e 3 \cdots \mathrm{N} 6^{\mathrm{i}} & 0.96 & 2.36 & 3.15(3) & 140\end{array}$

Symmetry codes: (i) $-x,-y,-z$; (ii) $-x, y+1 / 2,-z+1 / 2$; (iii) $x-1, y, z$; (iv) $x+1, y, z$; (v) $-x+1,-y,-z$; (vi) $-x+1, y+1 / 2,-z+1 / 2$; (vii) $x+1, y+1, z$. 